\title{
Ultrashort-pulse fiber ring lasers
}

\author{
L.E. Nelson*, D.J. Jones, K. Tamura**, H.A. Haus, E.P. Ippen
}

Department of Electrical Engineering, and Research Laboratory of Electronics, Massachusetts Institute of Technology, Cambridge, MA 02139, USA (E-mail: lenelson@mit.edu)

Received: 18 February 1997/Revised version: 22 April 1997

\begin{abstract}
This paper reviews recent progress on ultrashort pulse generation with erbium-doped fiber ring lasers. The passive mode-locking technique of polarization additive pulse mode-locking (P-APM) is used to generate stable, selfstarting, sub-500 fs pulses at the fundamental repetition rate from a unidirectional fiber ring laser operating in the soliton regime. Saturation of the APM, spectral sideband generation, and intracavity filtering are discussed. Harmonic modelocking of fiber ring lasers with soliton pulse compression is addressed, and stability regions for the solitons are mapped and compared with theoretical predictions. The stretchedpulse laser, which incorporates segments of positive- and negative-dispersion fiber into the P-APM fiber ring, generates shorter (sub-100 fs) pulses with broader bandwidths (> $65 \mathrm{~nm}$ ) and higher pulse energies (up to $2.7 \mathrm{~nJ}$ ). We discuss optimization of the net dispersion of the stretched-pulse laser, use of the APM rejection port as the laser output port, and frequency doubling for amplifier seed applications. We also review the analytical theory of the stretched-pulse laser as well as discuss the excellent noise characteristics of both the soliton and stretched-pulse lasers.
\end{abstract}

PACS: $42.60 F ; 42.65 ; 42.80$

Fiber lasers were made possible in the 1960s by the incorporation of trivalent rare-earth ions such as neodymium, erbium, and thulium into glass hosts [1]. Soon thereafter neodymium was incorporated into the cores of fiber waveguides [2,3]. Due to the high efficiency of the $\mathrm{Nd}^{+3}$ ion as a laser, early work focused on $\mathrm{Nd}^{+3}$-doped silica fiber lasers operating at $1.06 \mu \mathrm{m}$ [4]. Doping of silica fibers with $\mathrm{Er}^{+3}$ ions was not achieved until the 1980s [5,6]. Since that time $\mathrm{Er}^{+3}$-doped fiber lasers have received much attention, because the lasing wavelength at $1.55 \mu \mathrm{m}$ falls within the low-loss window of optical fibers and thus is suitable for optical fiber communications. Rare-earth ions such as $\mathrm{Ho}^{+3}[7,8], \mathrm{Tm}^{+3}$ [9-11], and

\footnotetext{
*Current address: Lucent Technologies/Bell Labs, 791 Holmdel-Keyport Road, Holmdel, NJ, USA

** Current address: NTT Access Network Systems Laboratory, Tokai-mura, Naka-gun, Ibaraki-ken 319-11, Japan
}

$\mathrm{Yb}^{+3}[12,13]$ have also been used as dopants or co-dopants in silica or fluoride fibers, allowing new lasing or pumping wavelengths, and $\mathrm{Pr}^{+3}$ has been incorporated into fluoride fiber, providing emission at $1.3 \mu \mathrm{m}[14,15]$.

Among the numerous advantages of fiber lasers are simple doping procedures, low loss, and the possibility of pumping with compact, efficient diodes. The fiber itself provides the waveguide, and the availability of various fiber components minimizes the need for bulk optics and mechanical alignment. Many different cavity configurations can be easily built with fibers and fused-fiber couplers, including linear FabryPerot, ring, and combinations of the two. Enhancement of the fiber nonlinearity due to large signal intensities and long interaction lengths is an additional advantage of fiber lasers that is particularly important for mode-locking. Potential applications for compact, diode-pumped, fiber-compatible optical sources at various wavelengths have motivated significant fiber laser research over the last 10 years.

In addition to offering continuous-wave operation, fiber lasers can be mode-locked to generate one or more pulses per round-trip. Mode-locked lasers have a number of potential applications, depending on the wavelength and pulse width. They could be used as sources in communications systems for time-division multiplexing (TDM) [16,17] or wavelength-division multiplexing (WDM) [18-20], as spectroscopic tools in the laboratory for time-resolved studies of fast nonlinear phenomena in semiconductors, or as seeds for solid-state amplifiers such as Nd:glass [21], color center [22], alexandrite [23], or Ti:sapphire. Short pulses also have potential use in electro-optic sampling systems, as a source for pulsed sensors, or as tunable seed pulses for lasers in medical applications. Applications such as optical coherence tomography could take advantage of the broad bandwidth of a mode-locked fiber laser rather than the temporal ultrashort pulse width.

\section{Review of passive mode-locking techniques and results}

Fiber lasers can be mode-locked actively, where a modulator produces amplitude or phase modulation, or passively, where an intensity fluctuation acts in conjunction with the 
fiber nonlinearity to modulate the cavity loss without external control. The upper-state lifetimes of rare-earth-doped fibers are slow $(\sim \mathrm{ms})$ implying that the gain does not react significantly within the cavity round-trip time $(<0.5 \mu \mathrm{s})$. A fast saturable absorber is therefore required to clean up both the leading and trailing edges of the pulse. Rare-earth-doped fiber lasers are also susceptible to Q-switching due to their long upper-state lifetimes. Passive mode-locking of fiber lasers to generate subpicosecond pulses has been achieved using three main methods: nonlinear amplifying loop mirror [24-26], nonlinear polarization rotation (also called polarization additive pulse mode-locking or Kerr mode-locking) [27-29], and semiconductor saturable absorbers [30-32].

Both the nonlinear amplifying loop mirror (NALM) and nonlinear polarization rotation rely on the Kerr effect in a length of optical fiber in conjunction with polarizers to cause artificial saturable absorber action and achieve pulse shortening. The NALM consists of a fiber Sagnac interferometer with gain placed asymmetrically in the loop. A differential phase shift occurs between the two directions in the NALM, and with proper phase bias the NALM transmits higher intensities while reflecting low intensities. The NALM is attached to a unidirectional fiber ring, forming a figure- 8 shaped cavity where the reflected low intensities are then extinguished by the isolator in the unidirectional ring. Pulses as short as $98 \mathrm{fs}$ have been generated at $1.57 \mu \mathrm{m}$ by an $\mathrm{Er}^{+3}$. doped fiber laser with an NALM and an $\mathrm{Er}^{+3}$-doped fiber amplifier in the unidirectional, linear loop [33]. A disadvantage of figure- 8 lasers has been the tendency for operation with bursts of multiple pulses because of long cavity lengths.

Various semiconductor saturable absorber structures have been incorporated into fiber lasers to obtain mode-locking with simple cavity designs. A strained superlattice was used in transmission in an $\mathrm{Er}^{+3}$-doped fiber ring laser [30], while a linear cavity with $\mathrm{Er}^{+3} / \mathrm{Yb}^{+3}$-doped fiber was modelocked with a nonlinear mirror consisting of an InGaAs/InP multiple-quantum-well (MQW) integrated on a Bragg reflector stack [31]. Desouza et al. [32] demonstrated self-starting mode-locking with polarization-maintaining (PM) fiber and a bulk InGaAsP sample on InP to obtain nearly transformlimited 320-fs pulses. Pulses of $2.3 \mathrm{~nJ}$ and $5.5 \mathrm{ps}$ were generated by an $\mathrm{Er}^{+3} / \mathrm{Yb}^{+3}$-doped fiber laser that suppressed multiple-pulse behavior at high pump powers with bulk and multiple-quantum well absorbers and a linear loss element [34]. Designs of saturable absorber structures that optimize the saturation characteristics include the anti-resonant Fabry-Perot mirror (A-FPSA), which was used to obtain selfstarting 260-fs pulses in a $\mathrm{Nd}^{+3}$-doped fiber laser [35], and the saturable Bragg reflector, which generated 235-fs pulses at fundamental repetition rates up to $200 \mathrm{MHz}$ in a short $\mathrm{Er}^{+3} / \mathrm{Yb}^{+3}$-doped fiber laser [36]. Improved understanding of the nonlinear dynamics of these structures permits optimization of different laser geometries and is the subject of current research.

Nonlinear polarization rotation relies on the intensitydependent rotation of an elliptical polarization state in a length of optical fiber. With proper settings of the initial polarization ellipse and phase bias, pulse shortening occurs. Mode-locking has been achieved using this technique in linear cavities with $\mathrm{Nd}^{+3}$-doped fiber [27] and $\mathrm{Er}^{+3}$-doped fiber [37], although these lasers required active modulators for pulse initiation. Simulations of a soliton fiber ring laser showed that a saturable absorber (nonlinear polarization rotation) and frequency limiter were required for the ring laser to self-start [38]. A self-starting ring cavity with low-birefringence fiber was then demonstrated at $1.55 \mu \mathrm{m}$, although the 1.2-ps soliton pulses were randomly spaced in bunches at the round-trip frequency [28]. Stable, self-starting, sub-500 fs soliton pulses at the fundamental repetition rate were first demonstrated in a short unidirectional ring [29]. Modifications of these initial experiments have resulted in significant improvements in mode-locked performance. A scheme for environmental stability was introduced in [39], and self-starting of a linear cavity was aided by a semiconductor saturable absorber mirror [40]. Diode pumping and addition of a birefringent tuning plate to the ring laser of [29] allowed tuning ranges as wide as $50 \mathrm{~nm}$ centered at $1.55 \mu \mathrm{m}$ [41]. Shorter and higher power pulses have been achieved using the stretched-pulse technique with nonlinear polarization rotation [42] and employing the rejection port as the output port [43], as discussed in Sect. 4. The stretched-pulse technique has yielded the shortest, broadestbandwidth pulses from an $\mathrm{Er}^{+3}$-doped fiber laser (63 fs) [44], while 2.7-nJ, 100-fs pulses have also been achieved [45]. With no practical limitations on pulse width or wavelength of operation, nonlinear polarization rotation in a fiber ring laser geometry offers the potential for short pulses and (or) wide tunability.

Although much work has focused on mode-locking of $\mathrm{Er}^{+3}$-doped fiber lasers due to the $1.55 \mu \mathrm{m}$ wavelength, employment of these mode-locking techniques with other rareearth doped fibers has resulted in the demonstration of subpicosecond pulses at additional infrared wavelengths. $\mathrm{Nd}^{+3}$ doped fiber lasers at $1.06 \mu \mathrm{m}$ have been mode-locked with nonlinear polarization rotation and with saturable absorber mirrors. Pulses as short as 42 fs were generated from nonlinear polarization rotation started by a moving mirror, where the positive group velocity dispersion of the fiber was compensated by a dispersive delay line [46]. Higher-power, soliton pulses were obtained with a chirped-fiber grating and MQW saturable absorber mirror at $1.06 \mu \mathrm{m}$ [47]; and a tuning range of $75 \mathrm{~nm}$ with femtosecond pulses was obtained with an optimized MQW in a $\mathrm{Nd}^{+3}$-doped fiber laser [48]. Additional references to work on mode-locked $\mathrm{Nd}^{+3}$-doped fiber lasers may be found in the paper by Fermann et al. in this issue. Work with $\mathrm{Pr}^{+3}$-doped fluoride fiber lasers at $1.3 \mu \mathrm{m}$ has employed the NALM in figure- 8 cavities to generate 1.6 -ps pulses [49] and $620-\mathrm{fs}$ pulses [50]. $\mathrm{A} \mathrm{Tm}^{+3}$-doped silica fiber laser was mode-locked with nonlinear polarization rotation and produced sub-500 fs pulses tunable from 1.8 to $1.9 \mu \mathrm{m}$ [51]. Mode-locking of a $\mathrm{Tm}^{+3}$-doped fiber laser with an MQW mirror has also been achieved with 190-fs pulses at $1.9 \mu \mathrm{m}$ [52]. The demonstration of fiber lasers as compact, practical sources of ultrashort pulses at a variety of wavelengths in the infrared is important for their possible application in communications, medicine, or as tools in the laboratory.

This paper reviews results obtained from mode-locked $\mathrm{Er}^{+3}$-doped fiber lasers employing nonlinear polarization rotation and soliton shaping in unidirectional rings. Polarization additive pulse mode-locking (P-APM) is described immediately below, followed by a discussion of self-starting in linear and ring cavities. Passively mode-locked fiber soliton lasers are the subject of Sect. 2. Although these soliton 
lasers have limited pulse widths and pulse energies, tunable operation across the entire $\mathrm{Er}^{+3}$ bandwidth can be achieved as discussed in Sect. 2.3. Harmonic mode-locking of fiber lasers is addressed in Sect. 3 with emphasis on pulse energy stabilization, frequency stabilization, and soliton pulse compression. Stretched-pulse lasers are the subject of Sect. 4, which includes a comparison of the soliton and nonsoliton (stretched-pulse) regimes of operation and pulse dynamics in the stretched-pulse laser. A formalism that describes this case where the pulse undergoes large changes per pass due to varying dispersion is reviewed. The optimization of the stretchedpulse laser for frequency doubling is discussed in Sect. 4.5 with use of frequency-resolved optical gating (FROG) to characterize the fundamental and frequency-doubled pulses. Finally, measurements and theory of the noise in fiber lasers mode-locked by P-APM are addressed in Sect. 5 .

\subsection{Polarization APM}

Additive pulse mode-locking (APM) is a passive modelocking technique that employs a nonlinear interferometer to achieve pulse shortening. The pulse is split into the two arms of the interferometer with a nonlinear element placed in one arm. The pulses recombine at the beam splitter, and pulse shortening occurs through the coherent addition of the selfphase modulated pulses. One advantage of APM is that it is extremely fast because it is based on self-phase modulation (SPM) from the Kerr effect in glass. Thus APM should not impose a practical limit on the shortest pulses. The technique has been extensively studied both experimentally and theoretically in several solid-state lasers [53-55], and the reader is encouraged to see these references for further details. APM has been extended to fiber lasers, where pulse shortening is achieved through SPM and polarization control.

Nonlinear polarization rotation can occur in an optical fiber when the initial polarization state is elliptical. The ellipse can be resolved into right- and left-hand circular polarization components of different intensities. These two circular components then accumulate different nonlinear phase shifts related to the intensity dependence of the refractive in$\operatorname{dex}\left(n=n_{0}+n_{2} I\right)[56,57]$. The polarization ellipse rotates while maintaining its ellipticity and handedness. An optical fiber is particularly well suited for nonlinear polarization rotation because the small mode diameter leads to high intensities, and thus to a large nonlinear index change, and because

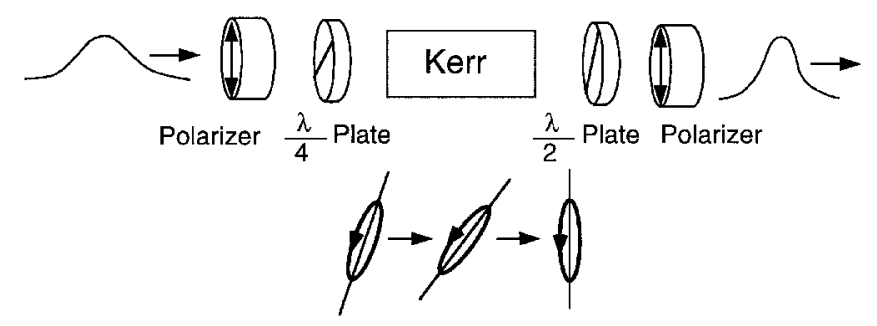

Fig. 1. Diagram of how pulse shortening occurs in a laser through P-APM. An initial pulse is linearly polarized and then made elliptically polarizated with a quarter-wave plate. The light then passes through an optical fiber where ellipse rotation occurs and the peak of the pulse rotates more than the pulse wings. At the output of the fiber, the half-wave plate orients the pulse so that the peak of the pulse passes through the polarizer while the wings of the pulse are extinguished, thus achieving pulse shortening long fiber lengths can be used. Figure 1 shows how nonlinear polarization rotation can be used in conjunction with bulk polarization optics to obtain an artificial saturable absorber (an element that absorbs low intensities but is bleached by and transmits high intensities) and to mode-lock the laser. The mode-locking technique is called polarization additive pulse mode-locking (P-APM) since the right- and left-hand circular polarization components acquire a differential nonlinear phase shift and are added together at the final polarizer.

\subsection{Ring configuration for self-starting}

Ideally, a passively mode-locked laser will evolve into a pulsed state on its own, without an external perturbation or trigger. This is called self-starting, meaning that the pulses start up from an initial noise fluctuation formed by mode beating of the multiple axial modes in the laser. In general, systems mode-locked by fast saturable absorbers have difficulty with self-starting due to the weak pulse shaping for long pulses [58,59]. Random mode-beating fluctuations decay within a characteristic time (the lifetime of the fluctuation) due to competing scattering processes [60]. A mode-locked state can only be established if the excess round-trip gain experienced by the initial fluctuation is large enough to complete the mode-locking process within the lifetime of the fluctuation. The lifetime is related to the linewidth of the first beat note of the power spectrum of the free-running laser [60].

Lasers in a unidirectional ring configuration have been shown to self-start more easily, as explained theoretically in references [61] and [62]. Both papers point out that mode pulling will decrease the mode coherence time (decreasing the lifetime of a fluctuation). References $[61,63]$ explain that spurious reflections (off bulk elements or polished fiber ends) and etalons in the cavity create a multiple Fabry-Perot structure with unevenly spaced axial modes. Larger injection signals (higher powers) are then needed to start the pulses. In a linear cavity, a single reflection surface can cause mode pulling. In a unidirectional ring cavity, though, the effect of reflections is reduced, since two reflections must occur in order to form an etalon. (A first-order backward reflection is attenuated in the isolator.) As reported in [61], low selfstarting thresholds appear to be possible for a ring cavity, whereas significantly more power (a factor of $\sim 10$ to 100 ) may be required for self-starting in a linear cavity.

Spatial hole burning in the gain medium, which occurs in a linear cavity even in the absence of spurious reflections, is also a strong inhibitor of self-starting [62]. The forward and backward propagating waves form a standing-wave pattern of the electric field and induce a spatial modulation of the complex refractive index in the gain medium. This grating, internal to the cavity, then causes mode pulling. Consequently, the self-starting threshold is inherently higher for linear cavities than for unidirectional ring cavities. The deleterious effects of spurious reflections in ring cavities are, of course, exacerbated by spatial hole burning. Reference [62] also points out that since there is a finite buildup time for the population grating in the gain medium, sudden perturbations or periodic variations of the cavity length can partially erase the grating and/or align the phases of three or more modes and enable self-starting. This explains why moving mirrors or "tapping the optical table" are helpful starting mechanisms. The en- 
hanced self-starting of the unidirectional ring is an important advantage of this laser configuration.

\section{Fiber soliton lasers}

The first all-fiber ring cavity to produce stable subpicosecond pulses at the fundamental repetition rate is shown in Fig. 2 [29]. The polarizer at the output of the fiber-pigtailed isolator and the polarization controller set the elliptical polarization state, which then rotates in the fiber due to the Kerr nonlinearity. The polarizer at the isolator input then transforms the rotation of the ellipse into amplitude modulation. A second polarization controller at the isolator input is used to compensate for any residual birefringence in the non-PM fiber and would not be necessary if the fiber were perfectly isotropic.

The pulse in such a laser can be described using a steadystate analysis that treats mode-locking in the time domain, as in [55]. The method analyzes the action of each component of the laser system on the pulse and imposes selfconsistency after one round-trip. Self-amplitude modulation (SAM) models the saturable absorber action due to P-APM. SAM is the important component in producing mode-locking, but the changes it produces in one round-trip are assumed small $(\sim 10 \%)$ in this analysis. We also assume that the mode-locked pulse experiences only small changes per pass (linear and nonlinear). The electric field for the radiation inside the laser cavity has the form:

$E(t)=u(t) \exp \left(j \omega_{0} t\right)$

where $u(t)$ is the complex amplitude of the slowly varying temporal envelope of frequency $\omega_{0}$. The Master equation [55] takes into account the possible effects in a mode-locked laser:

$$
\left\{-\jmath \psi+g-\ell+\left(\frac{g}{\Omega_{g}^{2}}+\jmath D\right) \frac{\partial^{2}}{\partial t^{2}}+(-\jmath \delta+\gamma)|u|^{2}\right\} u=0,
$$

where $\psi$ is the net linear phase shift, $\ell$ is the linear loss per pass, and $g$ is the laser gain. The gain is assumed to be slow, saturable gain, meaning that as the pulse energy increases, the gain saturates but on a timescale much longer than the pulse width. The second term proportional to $g$ is due to the finite bandwidth of the gain. $D=\frac{1}{2} k^{\prime \prime} L_{\mathrm{D}}$ represents the contribution of the group velocity dispersion (GVD) $k^{\prime \prime}$ in a material

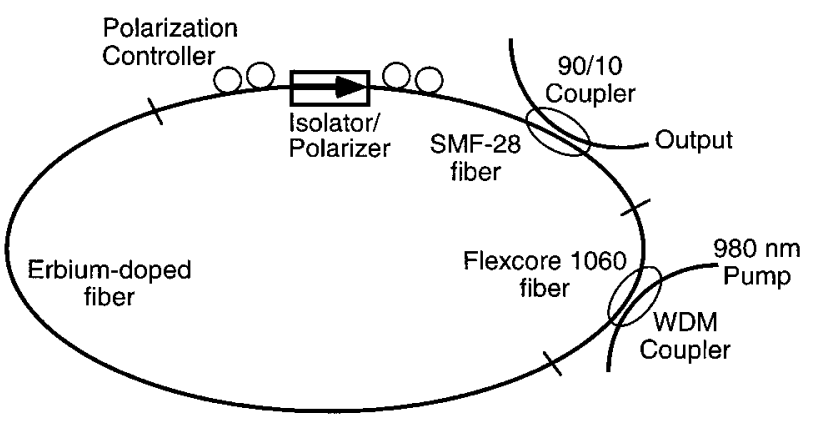

Fig. 2. Schematic of all-fiber ring laser mode-locked by P-APM, from [29]. WDM: wavelength-division-multiplexed coupler of length $L_{\mathrm{D}}$, while $\delta$ is the SPM contribution of the Kerr medium $\left[\delta=2 \pi n_{2} L_{\mathrm{K}} /\left(\lambda A_{\text {eff }}\right)\right.$ where $L_{\mathrm{K}}$ is the length of the Kerr medium, $n_{2}$ is the nonlinear index, $\lambda$ is the wavelength, and $A_{\text {eff }}$ is the effective mode area]. The last term on the right-hand side represents the SAM, where $\gamma$ is inversely proportional to the loss saturation intensity and must be positive so that higher intensities see lower loss.

The Master equation has an exact solution, as previously recognized by Martinez et al. [64, 65]:

$u(t)=A_{0}\left[\operatorname{sech}\left(\frac{t}{\tau}\right)\right]^{(1+\jmath \beta)}$,

where $\tau$ is the normalized pulse width $\left(\tau=0.567 \tau_{\mathrm{FWHM}}\right)$ and $\beta$ is the chirp parameter. In the negative GVD regime, it is possible to obtain chirp-free pulses $(\beta=0)$, such that the contributions from SPM and GVD balance, while the filtering and SAM also balance. The mode-locked pulse is then transform-limited and has the same secant-hyperbolic pulse shape as a soliton. Although the pulses are sech-like, they do not behave as solitons within the components of the system [66]. We can view the pulses as solitons only by averaging over the system operation such that the pulses are "average solitons", similar to those occurring in periodically amplified transmission systems $[67,68]$. Although the attenuation and amplification within one round-trip can be considerable, an important requirement on these "solitons" is that the nonlinear phase shift per round-trip must be small $(\ll 2 \pi)$, as will be explained in Sect. 2.2.2.

The Master equation as expressed in (2) considers the effects in the mode-locked laser as perturbations and does not guarantee stability of the pulse solutions in all limits. In fact, as pointed out in [69], energy fluctuations will grow exponentially without gain saturation to counterbalance the APM action. In solid-state lasers with slow, saturable gain and instantaneous SAM, we would expect some perturbations to grow too quickly for the pulse to be stabilized by gain saturation. Because stable pulses are observed experimentally, another effect in the laser must be providing stability. This effect can be represented in the Master equation by a fifth-order saturable absorber term $\left(\gamma_{5}|u|^{4} u\right.$, where $\left.\gamma_{5}<0\right)[70]$. In the fiber laser $\gamma_{5}$ is the result of saturation of the APM and the generation of spectral sidebands, as explained in more detail in Sect. 2.2. This fifth-order saturable absorber term causes the solutions of the Master equation to be more complicated than the hyperbolic-secant solution of (3). The $\gamma_{5}$ term was not included in the analysis of [55] because it is responsible for pulse stabilization and does not contribute significantly to the formation of pulses from a continuous-wave (cw) state.

Reference [66] presents an analysis that treats the fiber ring laser as a nonlinear Mach-Zehnder interferometer, where the right- and left-hand circular polarizations are the two arms of the interferometer. The polarizer provides one output port of the interferometer, while the other output port represents the absorbed polarization. The intensity-dependent interference at the Mach-Zehnder output then represents the intensity-dependent loss that occurs in the mode-locked laser. A matrix formulation is used to evaluate the loss parameter $\ell$, SAM parameter $\gamma|u|^{2}$, and SPM parameter $\delta|u|^{2}$ in terms of the the nonlinear phase shifts for the circular polarizations and the initial polarization ellipse. By adjusting the initial po- 
larization, the ratio $\gamma / \delta$ can be maximized, thus optimizing pulse width, chirp, and stability, as discussed in [55].

\subsection{Experimental results}

The laser shown in Fig. 2 had a total length of 4.8 meters of fiber in the ring and was pumped with a cw Ti:sapphire laser at $980 \mathrm{~nm}[29]$. The $\mathrm{Er}^{+3}$-doped fiber had a concentration of $1000 \mathrm{ppm}, N A$ of 0.13 , and mode-field diameter of $8.1 \mu \mathrm{m}$. Mode-locked operation was obtained with $50 \mathrm{~mW}$ of pump and $240 \mu \mathrm{W}$ average output power from the $10 \%$ output coupler. The pulses were self-starting and operated with a stable single pulse per round-trip. By increasing the pump power, the output power could be increased to $384 \mu \mathrm{W}$ before multiple pulse operation ensued at $85 \mathrm{~mW}$ of pump power. Figure 3 a shows an autocorrelation of a 450 -fs pulse (assuming a sech profile) for single-pulse operation with an output power of $192 \mu \mathrm{W}$ [29]. With an average dispersion of $-19 \mathrm{ps}^{2} / \mathrm{km}$ for the fiber in the ring, the theoretical peak soliton power for the laser was $\sim 110 \mathrm{~W}$ with a 450 -fs pulse, which was close to the measured value. The corresponding spectrum is shown in Fig. $3 \mathrm{~b}$ and had an approximate spectral width of $9 \mathrm{~nm}$ (assuming sech) with time-bandwidth product (TBP) of 0.54 .
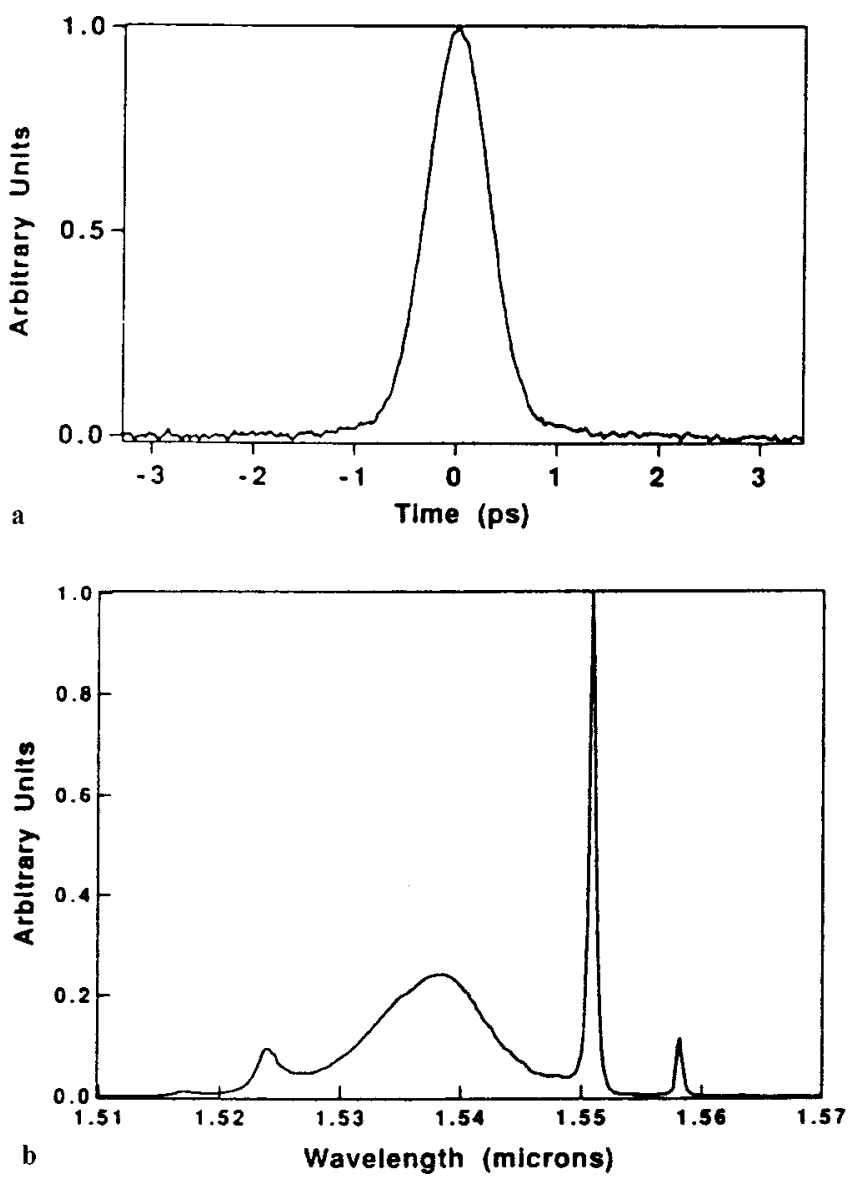

Fig. 3. a Autocorrelation of a 450-fs pulse from the all-fiber soliton ring laser. b Corresponding spectrum centered at $1535 \mathrm{~nm}$ with approximate spectral width of $9 \mathrm{~nm}$, from [29]

\subsection{Limitations of soliton lasers}

2.2.1 Saturation of APM. The operation of the all-fiber soliton laser points to several inherent limitations of the soliton regime. A tendency for multiple-pulse operation with increased pump power is due to quantization of soliton pulses in a system with excessive gain [66]. The soliton area theorem states that the product of the peak amplitude $A_{0}$ and pulse width $\tau$ is fixed by the average dispersion and nonlinearity:

soliton area $=A_{0} \tau=\sqrt{\frac{2|D|}{\delta}}$.

The energy of a soliton, $W=2\left|A_{0}\right|^{2} \tau$, is thus limited when either the peak power or pulse width is limited. Although the SAM (APM action) is generally represented by $\gamma|u|^{2}$ as in (2), it may be more accurate to represent it by $\sin \left(\gamma|u|^{2}\right)$ since the APM action is interferometric. A pulse passing through this APM structure has an energy gain of

$\int_{-\infty}^{\infty} \mathrm{d} t|u|^{2} \sin \left(\gamma|u|^{2}\right)$,

with $u(t)=A_{0} \operatorname{sech}(t / \tau)$ as defined by (3). The maximum of (5) is found to be at

$\gamma\left|A_{0}\right|^{2} \simeq 0.6 \pi$

Now the peak power of the soliton is limited, implying a limited pulse energy from the area theorem. If the pump power is increased to obtain high powers, the peak power of the soliton will eventually reach the limit imposed by this APM saturation. Then the single pulse per cavity round-trip will break up into multiple, uncontrolled (untimed) pulses. Such pulse energy quantization has been observed and reported in figure- 8 lasers $[71,72]$ and in a long ring laser [28]. The all-fiber soliton laser of [29], however, could operate with a stable, single pulse per round-trip. This was accomplished by making the fiber loop as short as possible [to decrease $\gamma$ in (6)] and by adjusting the gain to be low enough so that the energy of one single quantized soliton would fully deplete the gain.

2.2.2 Spectral Sidebands. The mode-locked pulse spectrum of the all-fiber laser in Fig. 3b shows significant spectral structure related to the soliton-like nature of the pulses. Work in long-distance soliton transmission [67,73] and in fiber soliton lasers [74-77] has shown that a resonant instability exists when the period (the amplifier spacing or the laser length) $Z_{\mathrm{p}}$ approaches $8 Z_{0}$, where $Z_{0}$ is the soliton period and $8 Z_{0}=4 \pi \tau^{2} /\left|k^{\prime \prime}\right|$. This can be explained by a simple phase-matching argument. When a soliton propagates in a fiber laser, it encounters various periodic perturbations such as gain, filtering, and loss due to splices or output couplers. The perturbed soliton sheds dispersive radiation as it is reshaped back into a soliton. These linear dispersive waves are generated over the broad spectrum of the soliton and have a dispersion relation:

$k_{\text {lin }}=-\frac{\left|k^{\prime \prime}\right|}{2} \Delta \omega^{2}$, 
where $\Delta \omega$ is the frequency offset from the peak of the soliton spectrum.

Each frequency component then propagates at its own phase velocity. The dispersive waves generated each period $Z_{\mathrm{p}}$ will interfere destructively except at frequencies that are phase-matched. This phase-matching condition is expressed by

$Z_{\mathrm{p}}\left(k_{\mathrm{s}}-k_{\text {lin }}\right)=2 \pi m$,

where

$k_{\mathrm{s}}=\frac{\left|k^{\prime \prime}\right|}{2 \tau^{2}}$

is the propagation constant of the soliton, $m$ is an integer, and $Z_{\mathrm{p}}$ is the perturbation length (length of the laser). The phase-matching condition is shown in Fig. 4. By using (7) in the above relation, we can solve for the frequency offset $\Delta \omega$ where phase-matching occurs $[75,77]$ :

$\Delta \omega= \pm \frac{1}{\tau} \sqrt{m \frac{8 Z_{0}}{Z_{\mathrm{p}}}-1}$.

At these phase-matched frequencies, the dispersive radiation builds up and causes sidebands of order $m$ on the pulse spectrum (Fig. 4). For long pulses, $8 Z_{0}=4 \pi \tau^{2} /\left|k^{\prime \prime}\right|$ is large, so the sidebands are located far from the peak of the soliton. If the average dispersion and laser length are kept constant as the pulse width decreases, then $Z_{0}$ will decrease, and the sidebands will be located closer to the peak of the soliton. The resonant instability occurs when the pulse width is such that $Z_{\mathrm{p}}=8 Z_{0}$ and the frequency offset of the sideband $\Delta \omega=0$. Note that the amount of continuum that is generated at a certain frequency is proportional to the spectral amplitude of the soliton at that frequency [75]. The sidebands appear as a pedestal under the pulse and can contain an energy comparable to the pulse energy.

Although it might appear that the resonant instability could be avoided by decreasing the average dispersion $\left|k^{\prime \prime}\right|$ as

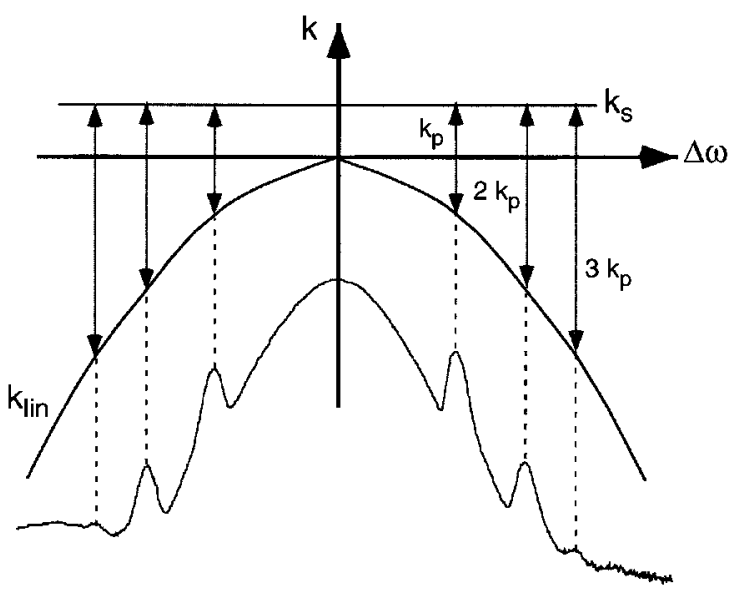

Fig. 4. Plot of the propagation constant $k$ versus angular frequency $\Delta \omega$ that shows the phase-matching condition for sidebands occurring when the pulse is periodically perturbed. The soliton-like pulse has a constant $k_{\mathrm{S}}$, while the dispersive waves have $k_{\text {lin }}$ with a quadratic dependence on $\Delta \omega$. Phase matching occurs at the particular frequencies where the periodic perturbation (with $k_{\mathrm{p}}$ ) makes up the difference between $k_{\mathrm{s}}$ and $k_{\text {lin }}$ the pulse width $\tau$ decreases, the soliton energy is proportional to $\left|k^{\prime \prime}\right|$, so this is not an optimal solution. Experimentally it has been found that the shortest pulses satisfy the condition $Z_{\mathrm{p}} \leq 3 Z_{0}$ in mode-locked $\mathrm{Er}^{+3}$-doped fiber lasers [78]. This implies that for the $\mathrm{Er}^{+3}$-doped fiber soliton laser, the total nonlinear phase shift per pass is limited to $3 \pi / 2$. The limited nonlinear phase shift leads to small peak powers and long pulse widths, and thus low pulse energies. For the $450-\mathrm{fs}$ pulse and spectrum shown in Fig. 3, the net cavity dispersion was $-0.091 \mathrm{ps}^{2}$. The distance $8 Z_{0}$ was then $\approx 50 \mathrm{~m}$, which was much longer than the loop length of $4.8 \mathrm{~m}$, indicating that the laser was operating far from the instability point [29].

The continuum due to periodic perturbations and the resulting sideband creation are important limitations on all soliton lasers. For a fixed cavity dispersion, the continuum generation limits the shortest obtainable pulse width, which in turn limits the pulse peak power due to the soliton area theorem. A small peak power then implies low average output power for operation with a single pulse per round-trip. Even without the pulse-width limitation imposed by periodic perturbations, a soliton laser would eventually have a limited pulse peak power due to the finite bandwidth of the gain material. Once the shortest pulse width is reached $\left(\tau_{\min } \propto 1 / \Delta v_{\text {gain }}\right)$, the peak power is fixed by the area theorem, implying limited pulse energies. But, generally the limit due to periodic perturbations occurs before the gain bandwidth becomes important.

\subsection{Filtering for sideband reduction and tuning}

Although it is not possible to avoid the nonlinear phase shift limit in soliton lasers, intracavity filtering can reduce the spectral sidebands without increasing the pulse width as shown by Tamura et al. [78] in a diode-pumped $\mathrm{Er}^{+3}$-doped fiber laser. The laser had a configuration similar to Fig. 2 except the fiber-pigtailed isolator and polarization controllers were replaced by bulk elements, and a polarizing beam splitter (PBS) and quartz plate of $5.12 \mathrm{~mm}$ thickness were added before the isolator. The PBS, quartz plate, and input polarizer of the isolator then comprised a Lyot filter with $\sim 23 \mathrm{~nm}$ bandwidth and $\sim 46 \mathrm{~nm}$ free spectral range. The authors reported reduced continuum by an order of magnitude with almost complete elimination of the spectral sidebands and no increase in pulse duration. With no filtering, the pulse width was $349 \mathrm{fs}$, while with filtering the shortest pulse was $311 \mathrm{fs}$. The birefringent filter also allowed broad tunability across the $\mathrm{Er}^{+3}$ gain with $43 \mathrm{~nm}$ of continuous tuning by adjustment of the tuning plate and pump power.

Filter optimization was reported in [41], where filters with varying bandwidths were used. Sidebands were reduced such that the ratio of the peak sideband to peak of the soliton spectrum was reduced to $<-17 \mathrm{~dB}$ for a $19.2-\mathrm{nm}$ filter. TBP were reduced from a range of 0.38 to 0.47 when spectral sidebands were large to a range of 0.34 to 0.38 when the sidebands were reduced, indicating that the pulse was slightly chirped when sidebands were present. The authors also reported that when the pulse width was limited by filtering, the spectral width was approximately one-half the filter bandwidth. Thus, to obtain maximum sideband reduction with minimal pulse broadening, a filter of approximately twice the bandwidth of the unfiltered spectrum should be used. A larger continuous tuning range of $50 \mathrm{~nm}$ was made possible by using 
a dual-plate filter that consisted of quartz plates of thicknesses $6.14 \mathrm{~mm}$ and $3.07 \mathrm{~mm}$ and provided a bandwidth of $19.2 \mathrm{~nm}$ and free-spectral range of $>80 \mathrm{~nm}$ [41].

\section{Harmonic mode-locking of fiber lasers}

Actively mode-locked fiber lasers are leading candidates for sources in optical communications systems because highquality pulses centered at $1.55 \mu \mathrm{m}$ can be locked to a master clock at high repetition rates $(>1 \mathrm{GHz})$ with low timing jitter. Mode-locking is generally achieved using a high-speed intracavity electro-optic modulator, and typical cavity lengths are usually $\geq 1 \mathrm{~m}$ due to the limited doping concentrations of $\mathrm{Er}^{+3}$ in silica fiber. Accordingly, to obtain a high pulse repetition rate, the modulator must be driven at a harmonic of the round-trip cavity frequency to produce multiple pulses in the cavity. If the fundamental cavity frequency is $v_{\mathrm{c}}$ and the modulation frequency is $v_{\mathrm{m}}=N v_{\mathrm{c}}$, the laser is mode-locked at the $N$ th harmonic with $N$ pulses in the cavity. This means that an axial mode is locked to every $N$ th mode falling within the gain bandwidth, forming a so-called supermode [79].

\subsection{Pulse energy stabilization}

Actively mode-locked fiber lasers were first demonstrated using $\mathrm{Nd}^{+3}$-doped fiber [80, 81] and $\mathrm{Er}-\mathrm{Yb}$-doped fiber [82] with pulse widths $\geq 70 \mathrm{ps}$ at the fundamental cavity repetition rate. Harmonic mode-locking was achieved by $[83,84]$, but the lasers suffered from pulse-to-pulse energy fluctuations. This unfortunate result is caused by the slow $(\approx 10 \mathrm{~ms})$ relaxation time of the $\mathrm{Er}^{+3}$ gain, which does not respond rapidly enough on the timescale of the pulse spacing $(\approx 1 \mathrm{~ns})$ to stabilize pulse energy variations. Pulse energy fluctuations are equivalent to a simultaneous oscillation of more than one supermode and can be diagnosed by examining the RF spectrum of the pulse train. A supermode suppression of greater than $45-50 \mathrm{~dB}$ is generally considered acceptable for optical communications sources. Bit-error-rate testers can also be used to examine the uniformity of the pulse trains.

Stabilization of pulse energies is clearly required before harmonically mode-locked fiber lasers can be used in optical communication applications. One solution is the introduction of a subcavity with a free spectral range that matches the modulation frequency $[85,86]$. Energy stabilization is achieved because a portion of each pulse in injected into successive pulses, injection locking the pulse train optically. The major disadvantage with this method is that it requires interferometric stabilization of the subcavity with respect to the modulation frequency.

A second, more appealing approach uses a passive, fast intensity-dependent loss mechanism. In this technique, an intracavity nonlinear element causes more intense pulses to experience higher loss. As long as the nonlinearity can recover to its initial state before the next pulse arrives (i.e. it is fast), the pulse intensities become clamped to a common value.

The first application of this technique used nonlinear polarization rotation [87]. As described above in Sect. 1.1, APM in fiber lasers uses nonlinear polarization rotation to create an artificial fast saturable absorber. By suitable adjustment of the APM (interference) bias, it is possible to impose intensity limiting on the pulses or additive pulse limiting (APL). APL was demonstrated using a laser similar to that shown in Fig. 2 but with a fiber-pigtailed Ti:LiNbO 3 Mach-Zehnder waveguide modulator placed between the isolator and output coupler. After appropriate adjustment of the polarization controllers, a 1-GHz pulse train was generated with a supermode suppression of $\approx 50 \mathrm{~dB}$. APL is dependent on the nearly instantaneous Kerr effect, so it can be safely scaled to much higher repetition rates.

Recently, a second technique using SPM and spectral filtering to obtain a fast intensity-dependent loss was reported $[88,89]$. In this case a more intense pulse generates a broader spectrum through SPM. An intracavity spectral filter then causes a higher-intensity pulse (with wider spectrum) to experience greater loss than a lower-intensity pulse. In this manner an optimum pulse bandwidth, and thus a standard pulse intensity, is imposed on the pulse train. As this method does not rely on polarization effects, it can be used in PM fiber lasers.

\subsection{Frequency and cavity stabilization}

A remaining difficulty with harmonically mode-locked fiber lasers is thermal drift of the cavity length. With

$\frac{\partial n}{\partial T}=1.1 \times 10^{-5} \quad /{ }^{\circ} \mathrm{C}$

for silica fiber and a typical fiber length of $50 \mathrm{~m}$, the ring cavity length fluctuates by $0.55 \mathrm{~mm} /{ }^{\circ} \mathrm{C}$. If the modulation is at the $N=2000$ harmonic, this translates to a frequency fluctuation of $\approx 60 \mathrm{kHz} /{ }^{\circ} \mathrm{C}$ about an $8-\mathrm{GHz}$ modulation frequency. Accordingly, slight thermal variations are able to cause the pulses to lose synchronism with the modulator. A number of techniques have been reported to compensate for thermal drift.

The most obvious stabilization scheme is a dynamic adjustment of the cavity length [90]. A portion of the fiber in the cavity is wound around a piezoelectric drum. By driving the drum with a proper error signal, the cavity length is adjusted to keep the cavity frequency synchronous with the fixed modulator drive frequency. A second solution uses regenerative feedback where the modulation frequency is derived directly from the pulse train [91]. Any change in the cavity length automatically adjusts the modulation frequency to maintain pulse-modulator synchronism. In a third solution, the modulation frequency is fixed and the cavity dispersion is sufficiently high that the pulses can remain synchronous with the modulator by shifting in wavelength [92].

An alternate approach abandons the requirement of pulsemodulator synchronism [93]. Anomalous-dispersion fiber is used throughout the cavity in this fiber laser to provide strong soliton shaping, and the active mode-locking element is a phase modulator that is purposely detuned from a cavity harmonic. When mode-locked, the resulting pulse train has a repetition rate that deviates $15-30 \mathrm{kHz}$ from the modulation frequency. Asynchronous phase modulation produces a sinusoidal frequency shift [94], which normally would destroy any pulses. Solitons, however, are able to fight the frequency shift [95] and remain intact. 


\subsection{Soliton pulse compression}

Sources of subpicosecond pulses are being evaluated for two primary ultrahigh bit rate communications systems applications: time-division multiplexing (TDM) and wavelengthdivision multiplexing (WDM). Pulse widths of $\leq 1 \mathrm{ps}$ are inherently required in $100 \mathrm{Gbit} / \mathrm{s}$ TDM systems [17]. For a WDM architecture, the broad spectrum of a subpicosecond pulse can be used as a high-repetition-rate, spectrally-sliced source [20].

As determined by Kuizenga and Siegman [97], the pulse width produced by active mode-locking is inversely proportional to the geometric mean of the mode-locking frequency and the bandwidth of the medium (or filters) in the resonator. Using typical parameters of fiber lasers, this expression predicts pulses widths to be $10-50$ ps. Pulses generated from stabilized harmonically mode-locked fiber lasers confirmed this prediction [86].

In order to shorten the pulses from actively mode-locked fiber lasers, the fiber medium naturally invites the use of SPM [98] or a combination of SPM and negative GVD [64]. Pulse shortening with SPM was first demonstrated in an actively mode-locked $\mathrm{Nd}^{+3}$-doped fiber laser [99]. Soliton shaping was then demonstrated in an $\mathrm{Er}^{+3}$-doped fiber ring laser [83] and in a $\mathrm{Nd}^{+3}$-doped fiber laser [100]. Soliton shaping was also achieved in $\mathrm{Er}^{+3}$-doped fiber lasers with an InGaAsP semiconductor amplifier [101] and with phase modulators $[102,103]$. The results of [98] were extended by Kärtner et al. [104] (and indirectly in [105]) who showed theoretically that a sufficiently large amount of negative GVD should suppress the normal mode-locked state enough to allow formation of a solitary pulse that is considerably shorter than the width predicted by [97]. The central idea is that the soliton parameters of the cavity, rather than the modulator characteristics, determine the pulse width.

Successful shortening of actively mode-locked pulses by soliton compression must satisfy the following two conditions: (i) The solitons must be successfully re-timed on each round-trip; (ii) there must be discrimination between the soliton state and the normal actively mode-locked state. The former condition of pulse re-timing is a practical matter of maintaining synchronism between the modulator and the pulse train and is addressed in the previous section on cavity drift. Following the analysis in reference [105], the latter condition can be quantified by calculating the loss of the soliton and the loss of a fundamental Gaussian pulse, which is the eigensolution of the active mode-locking equation (with no nonlinearity) [97]. If the soliton state is to remain stable, the Gaussian pulse of the active mode-locker must possess a higher loss than that of the soliton. The resulting condition is

$$
\frac{\pi^{2}}{24} M \Omega_{\mathrm{m}}^{2} \tau^{2}+\frac{1}{3 L \Omega_{\mathrm{f}}^{2} \tau^{2}}<\operatorname{Re} \sqrt{\frac{M \Omega_{\mathrm{m}}^{2}}{2}\left[\frac{1}{\Omega_{\mathrm{f}}^{2} L}-j \frac{k^{\prime \prime}}{2}\right]},
$$

where $M$ is the modulation index per unit length, $\Omega_{\mathrm{m}}$ is the modulation frequency, $\tau$ is the soliton pulse width, $\Omega_{\mathrm{f}}$ is the filter bandwidth, and $k^{\prime \prime}$ is the average GVD over the total cavity length $L$ so that $L_{\mathrm{D}}=L$. On the left side is the loss experienced by the soliton, and on the right side is the loss of the Gaussian mode-locked pulse.
In addition, the modulation must not drive the soliton unstable. From soliton perturbation theory [69], energy fluctuations of the soliton are damped if

$$
\frac{\pi^{2}}{8} M \Omega_{\mathrm{m}}^{2} \tau^{2}<\frac{1}{L \Omega_{\mathrm{f}}^{2} \tau^{2}}
$$

After fulfilling these two conditions, the pulse width is now specified by the soliton condition,

$\tau=\frac{4|D|}{W \delta}$,

where $W$ is pulse energy, $D$ is the dispersion, and $\delta$ is the nonlinearity.

The fiber laser used to investigate soliton compression of actively mode-locked pulses is shown in Fig. 5 [96]. The laser is constructed entirely of PM fiber, including the WDM coupler and output coupler, to eliminate any nonlinear polarization rotation, thereby excluding any artificial saturable absorber action (APM/APL) that could contribute to the pulse shortening and soliton stability. The average dispersion $k^{\prime \prime}$ of the cavity is $-10 \mathrm{ps}^{2} / \mathrm{km}$. Approximately $500 \mathrm{~mW}$ of the 978-nm MOPA pump is coupled into the WDM. An open air section is included in the ring to facilitate the placement of different optical filters in the cavity. A fiber-pigtailed $\mathrm{LiNbO}_{3}$ waveguide amplitude modulator is the active mode-locking element.

With a 7.80-nm filter and the modulation strength adjusted to satisfy both (12) and (13), the pulse autocorrelation shown in Fig. 6a is obtained. The corresponding optical spectrum in Fig. 6b shows evidence of Kelly sidebands [77]. Replacing the 7.80-nm filter with a 16.7-nm filter clearly reveals the sidebands, thereby confirming the pulses to be solitons. The experimentally measured pulse width of $634 \mathrm{fs}$ at $5 \mathrm{Gbit} / \mathrm{s}$ represents a pulse width shortening by a factor of 4.4 over the standard activly mode-locked pulse width. This shortening factor is in good agreement with the prediction of [104] for the dispersion and filter bandwidth in the laser. These 634-fs pulses are the shortest actively harmonically mode-locked pulses employing soliton compression to be generated directly from a fiber laser.

The stability region for the solitons, which lies between the two curves of (12) and (13), is shown in Fig. 7a. For

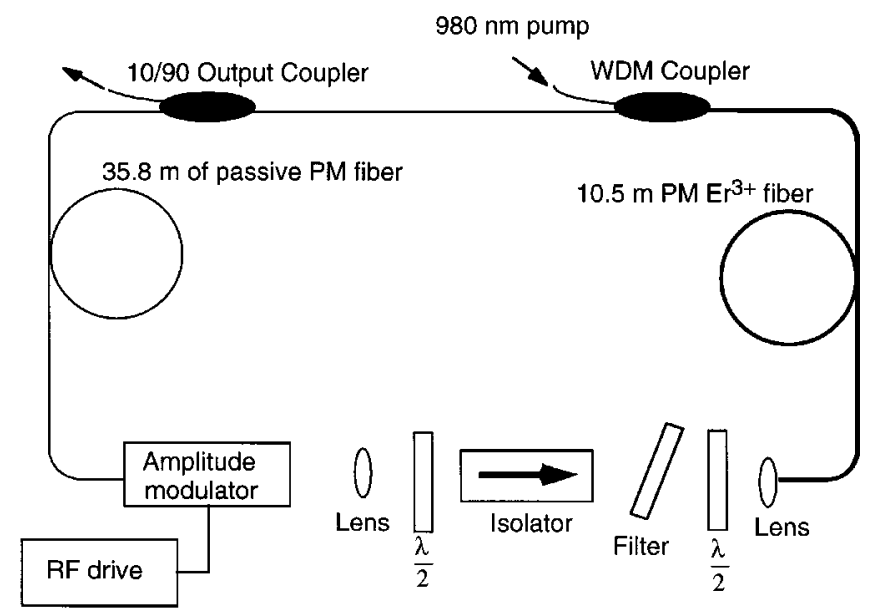

Fig. 5. Fiber laser using all-polarization-maintaining fiber from [96] 

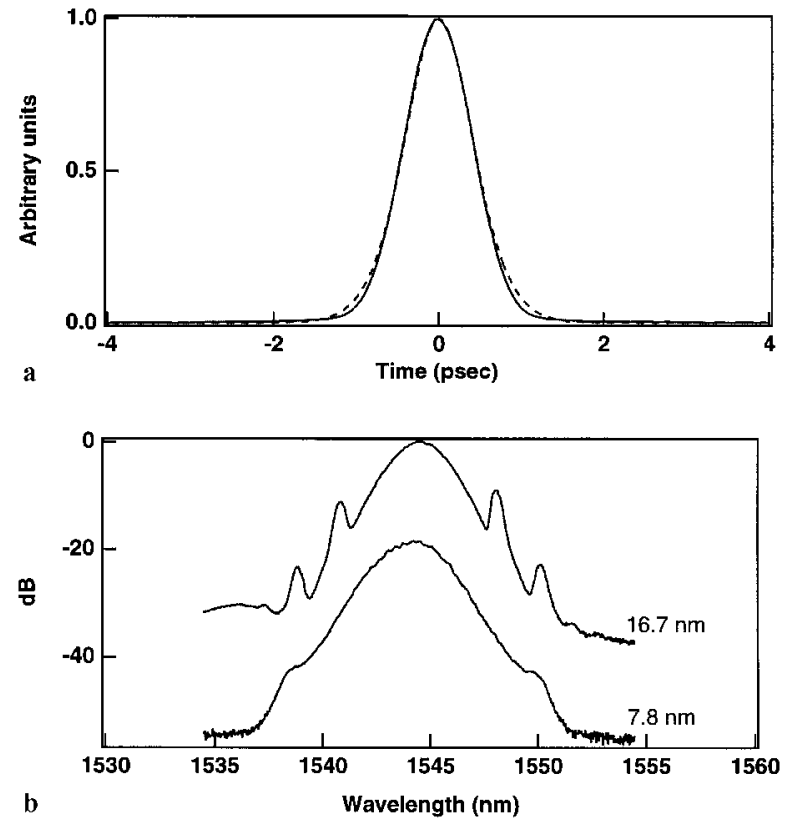

Fig. 6. a Autocorrelation trace of pulse from the actively mode-locked, allPM-fiber laser with a 7.8-nm intracavity filter. A sech fit (dashed curve) to the trace gives a FWHM pulse width of $634 \mathrm{fs}$. b Optical spectrum of corresponding pulse. Also shown is the optical spectrum with a 16.7-nm filter showing pronounced Kelly sidebands, confirming the pulse is indeed a soliton, from [96]

experimental observation of this regime, a series of different interference filters with varying bandwidths (5.47, $6.18,7.80,10.1,11.0,12.2$, and $16.7 \mathrm{~nm})$ were placed inside the cavity. The horizontal lines represent the explored regions at particular filter bandwidths. Autocorrelation traces for specific operating points, indicated by circles and squares, are shown in Fig. 7b. The circles represent operating points where clean solitons circulated in the cavity. No pedestal is observed on the autocorrelation trace at these points. The abscissa contains the soliton pulse width to the fourth power. Hence for each filter bandwidth we located each point on the plot by using the value of the modulation strength and experimentally observed soliton pulse width under constant cavity dispersion. The squares represent operating points that showed a mixture of Gaussian pulse shapes and solitons. These points are unstable according to the stability criterion predicted by (12) because there is no discrimination between the Gaussian pulse and the soliton.

The RF spectrum of the pulse train shows that, although the cavity modes are locked, supermode suppression is only 20-30 dB. As discussed in Sect. 3.1, this level of supermode suppression indicates variation of the pulse energies in the pulse train and is not acceptable for optical communications sources. In the present laser, soliton parameters serve to quantize the pulses, so poor supermode suppression actually means that the available gain is not sufficient to place a soliton in every available time slot. A calculation with the estimated intracavity power and the soliton energy indicates that approximately one-half of the time slots are filled with pulses. In fact, a bit pattern formed by occupied time slots (ones) and empty slots (zeros) can be observed on an oscilloscope and maintained indefinitely. This behavior can be exploited
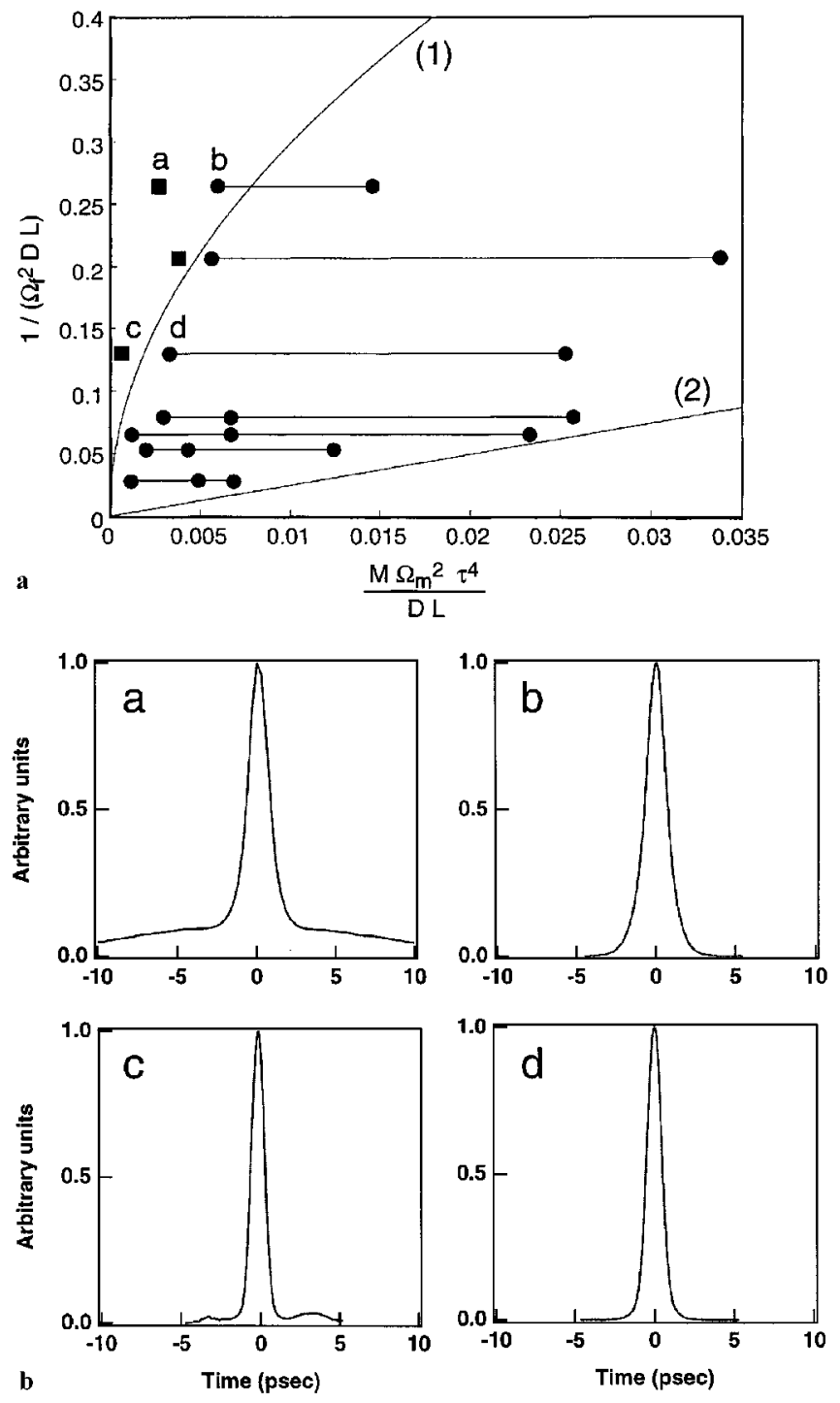

Fig. 7. a Stability region of solitons with the boundary curves (1) and (2) predicted by (12) and (13), respectively. The explored operating regions at different filter bandwidths are given by horizontal lines. The circles signify clean soliton operation. The squares indicate a mixture of solitons and Gaussian (mode-locked) pulses. b A series of autocorrelation traces referenced in Fig. 7a showing the transition between solitons and normal mode-locked pulses. Note the pedestals due to the competing Gaussian pulses in traces a and c, from [96]

to build an optical memory loop [106] that can store $3.8 \mathrm{kbit}$ packets at rates up to 50 Gbit per second [107, 108].

\section{Stretched-pulse laser}

As discussed in Sect. 2.2 soliton lasers have inherent practical limits on their pulse width and pulse energy due to spectral sideband generation and saturation of the APM. An approach to avoiding the soliton regime is to employ positive-dispersion fiber. Early reported systems minimized pulse shaping per pass by using short lengths of positive dispersion $\mathrm{Er}^{+3}$-doped fiber and prisms for negative dispersion. Pulses of $84 \mathrm{fs}$ with $10 \mathrm{pJ}$ of energy [109] and $190 \mathrm{fs}$ with $100 \mathrm{pJ}$ of energy [37] were obtained, but the linear cavities 


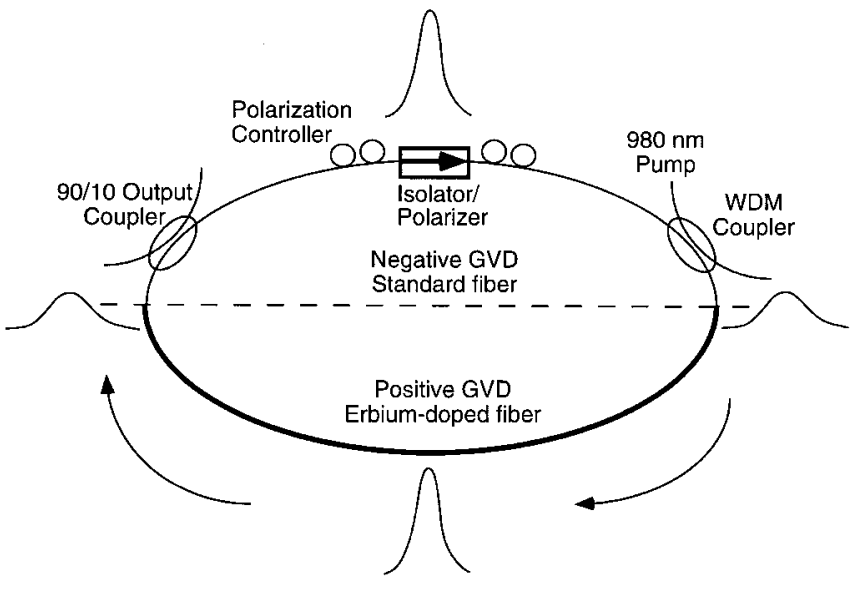

Fig. 8. Experimental setup for the all-fiber stretched-pulse laser from [42]

had bulk elements and were not self-starting. Then, Tamura et al. [42] introduced the stretched-pulse technique, where an all-fiber P-APM ring cavity is comprised of segments of alternately large positive- and negative-dispersion fiber with a net dispersion that is slightly positive, as shown in Fig. 8. In this laser the $\mathrm{Er}^{+3}$-doped fiber was $1.13 \mathrm{~m}$ and had an unpumped loss of $55 \mathrm{~dB} / \mathrm{m}$ at $1.535 \mu \mathrm{m}$, core diameter of $2.5 \mu \mathrm{m}, \delta n$ of 0.035 , and GVD of $+0.075 \pm 0.005 \mathrm{ps}^{2} / \mathrm{m}$. The Corning SMF-28 (standard) fiber had GVD of $-0.022 \mathrm{ps}^{2} / \mathrm{m}$. Self-starting pulses of $90 \mathrm{pJ}$ energy and $56 \mathrm{~nm}$ spectral width with durations as short as $77 \mathrm{fs}$ were obtained after chirp compensation in an external dispersive delay line of negativedispersion fiber.

In a stretched-pulse laser, the lengths of large positiveand negative-dispersion fiber cause the pulses to be alternately stretched and compressed as they circulate in the cavity, as shown in Fig. 8. The pulse width can change by an order of magnitude within the cavity, and thus the average peak power in the laser is lowered significantly. As a result, the net nonlinear phase shift per pass is lower, and APM saturation is avoided. Spectral broadening occurs in the doped fiber due to the action of the positive dispersion with SPM. At the output, the pulses can then have high energy and a broad bandwidth. Because the pulses are at a minimum for only a very small portion of the cavity round-trip, the nonlinear phase shift is limited, and the alternating dispersion is the dominant effect. Since the output is usually taken where the peak power is low, the output pulses have a large chirp, but one that is
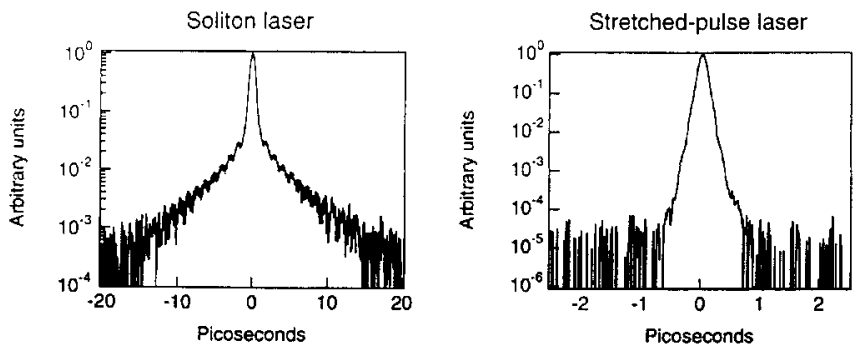

Fig. 9. Comparison of autocorrelation traces (plotted on a log scale) from the soliton fiber ring laser from [29] and stretched-pulse fiber ring laser with net dispersion of $+0.004 \mathrm{ps}^{2}$. Pulse widths were $450 \mathrm{fs}$ (assuming sech) for the soliton laser and $90 \mathrm{fs}$ (assuming Gaussian) for the stretched-pulse laser, from [110] highly linear [112]. The chirp can then be compressed with an external dispersive delay line composed of fiber, prisms, or gratings. Another important aspect of the stretched-pulse technique is that the alternating dispersion causes a periodically varying $k(\omega)$, which reduces phase-matched coupling to resonant sidebands [111]. Thus the spectra are cleaner and there is less dispersive radiation between pulses, as shown in Fig. 9 by the comparison of the soliton pulse autocorrelation from [29] with the stretched-pulse autocorrelation. The soliton autocorrelation shows an exponentially decaying pedestal due to dispersive wave radiation and beating of the first- and second-order sidebands.

\subsection{Soliton versus nonsoliton operation}

A systematic study of the effect of the stretched-pulse laser's net GVD on the output pulse characteristics was reported in [111]. Using a laser configuration similar to Fig. 8, the net dispersion $D_{\mathrm{T}}$ of the fiber ring was varied from $D_{\mathrm{T}} \approx$ $-0.09 \mathrm{ps}^{2}$ to $D_{\mathrm{T}} \approx+0.04 \mathrm{ps}^{2}$ by changing the positiveand negative-dispersion fiber lengths. (Compression of the positively chirped output pulses was done in the negativedispersion fiber of the output coupler, as in [42].) At large negative $D_{\mathrm{T}}$ the laser clearly operated in the soliton regime with spectral sidebands and with secant-hyperbolic spectra and autocorrelations. The stretching factors (ratio of maximum to minimum pulse width within the loop) were small $(\sim 3-5)$ and the pulse energies from the $10 \%$ output coupler were $<40 \mathrm{pJ}$. As the net dispersion was increased, the pulse widths decreased while the pulse energy increased, as shown in Fig. 10. The stretching factors also increased, and spectral sideband generation was reduced, indicating that improved operation can be obtained by stretching even when the net cavity dispersion is negative.

When $0 \leq D_{\mathrm{T}} \leq+0.04 \mathrm{ps}^{2}$, the pulses had spectral widths $>50 \mathrm{~nm}$ and large chirp. The stretching factors were between 10 and 20 for this range of $D_{\mathrm{T}}$, and the pulse autocorrelations and spectra were best fit to Gaussian profiles. The compressed pulses were in the range of $76 \mathrm{fs}$ to $105 \mathrm{fs}$, while the TBPs were in the range of $\sim 0.55$ to 0.65 . The shortest and cleanest pulses were observed for $D_{\mathrm{T}} \approx+0.011 \mathrm{ps}^{2}$ where the autocorrelation of the 76-fs pulse and corresponding spectrum are shown in Fig. 11. The spectral width was $64 \mathrm{~nm}$, corresponding to a time-bandwidth product of 0.60 , and the output

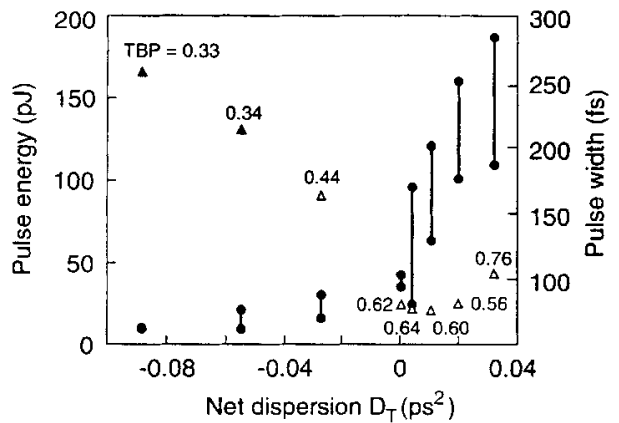

Fig. 10. Plots of pulse energy ranges (connected circles) and shortest FWHM pulse width (triangles with corresponding TBP next to each point) versus net dispersion $D_{\mathrm{T}}$ for the all-fiber stretched-pulse laser. Dark triangles assume sech, and open triangles assume Gaussian, from [111] 


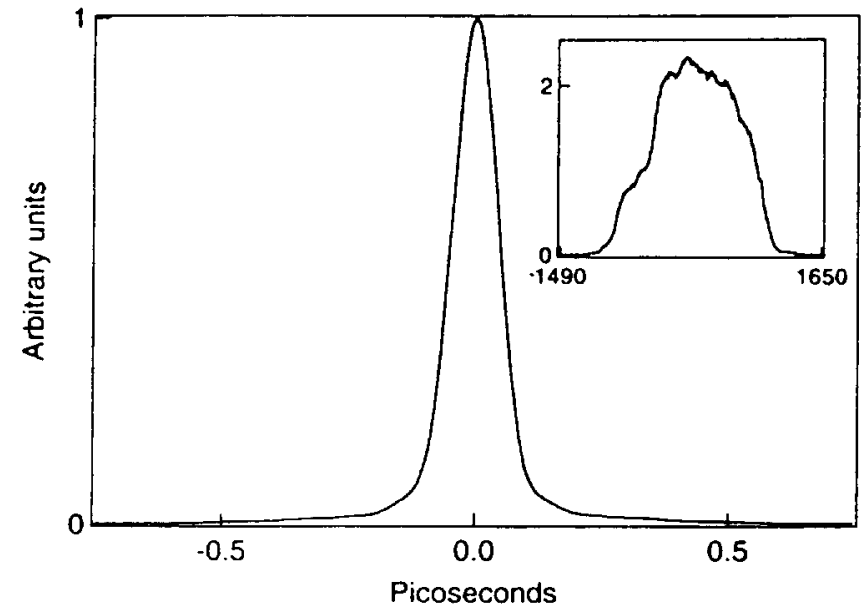

Fig. 11. Autocorrelation of 76-fs pulse (assuming Gaussian) and corresponding spectrum (inset) from the all-fiber stretched-pulse laser with net cavity dispersion $D_{\mathrm{T}}=+0.011 \mathrm{ps}^{2}$, from [111]

pulse energy (from the $10 \%$ output coupler) was $>100 \mathrm{pJ}$. For $D_{\mathrm{T}}>+0.02 \mathrm{ps}^{2}$ the pulse widths and TBPs increased, in part due to nonlinear chirp caused by higher intracavity powers, and possibly due to third-order dispersion. The experiments showed that the stretched-pulse laser has optimal output characteristics when the net cavity dispersion is slightly positive.

\subsection{Theory of stretched-pulse APM}

An analytical theory for stretched-pulse APM using a chirped Gaussian model and assuming small nonlinear changes per pass was described in [110]. The goal of the analysis was to formulate a Master equation, similar to (2), for the stretchedpulse laser. The theory asserts that, although the pulse changes greatly in one round-trip due to the GVD, the stretching and compressing is a linear, reversible process and is helpful in that it reduces the SPM per pass. Generally, pulses with clean spectra and good stability have small nonlinear changes per pass. The assumption of a chirped Gaussian pulse arises from the experimentally observed pulse shapes [111] and is justified on theoretical grounds as explained below.

The theory considers balanced linear dispersion as the dominant effect in the laser and treats the dispersion imbalance and nonlinearity as perturbations. An important aspect of the theory is the assumption of a transform-limited pulse in the symmetry plane of the ring with balanced dispersion, as indicated in Fig. 8. This assumption is based on the fact that having pulse-width minima at these locations minimizes the average width of the pulse and thus maximizes the nonlinear effects on the pulse. The nonlinear effects lead to APM action, and the maximization of the nonlinear effects maximizes the APM action. The loss of the laser is therefore minimized because APM action acts as an artificial saturable absorber that experiences maximum saturation when the two pulse-width minima occur at the symmetry plane.
The change of the pulse per pass due to the GVD imbalance is represented by the operator

${ }_{J} D \frac{\mathrm{d}}{\mathrm{d} t}=J\left(\frac{k_{p}^{\prime \prime} L_{p}}{2}-\left|\frac{k_{n}^{\prime \prime} L_{n}}{2}\right|\right) \frac{\mathrm{d}}{\mathrm{d} t}$,

where $k_{i}^{\prime \prime}$ is the GVD, $L_{i}$ is the fiber length, and the subscripts $p$ and $n$ denote positive and negative GVD, respectively. The accumulated nonlinear phase shift responsible for the SAM and SPM actions is evaluated using the zeroth-order approximation of Fig. 8 for the expanding and contracting pulse, which is transform-limited in the symmetry plane of the ring. It is clear that the temporal window of the SAM and SPM actions is now much wider than the pulse duration at the position of minimum pulse width. A Taylor series expansion of the pulse in time is terminated with the quadratic term leading to the Master equation:

$$
\begin{array}{r}
{\left[(g-\ell)+\left(\frac{g}{\Omega_{g}^{2}}+{ }_{j} D\right) \frac{\partial^{2}}{\partial t^{2}}+\gamma_{0}\left|A_{0}\right|^{2}\left(1-\mu \frac{t^{2}}{\tau_{0}^{2}}\right)\right.} \\
\left.-\jmath \delta_{0}\left|A_{0}\right|^{2}\left(1-\mu \frac{t^{2}}{\tau_{0}^{2}}\right)\right] u(t)=-\jmath \psi u(t),
\end{array}
$$

where $\mu<1$ is the curvature of the parabolic time dependence found either by expansion of the Gaussian solution or via a variational method, which gives better agreement with numerical solutions [113].

The Master equation has the Gaussian solution

$u(t)=A_{0} \exp \left(-Q \frac{t^{2}}{2}\right)$

where the complex parameter $Q$ is obtained by balancing terms in (16). The Gaussian pulse shape is an approximation to the actual pulse shape. The Taylor expansion of the SAM and SPM coefficients around the peak of the pulse is accurate near the center of the pulse, but fails in the wings. Hence, one expects the actual pulse shape to be Gaussian in the high-intensity portion of the pulse and to be exponential in the wings where the nonlinearity ceases to act on the pulse. This is confirmed by the autocorrelation trace of the stretched-pulse laser shown in Fig. 9.

The Gaussian solution of (16) has another important implication. A perturbation of the six pulse parameters, amplitude, phase, width, chirp, timing, and carrier frequency, is expressed in terms of the three lowest-order Hermite Gaussians with complex coefficients. These are all bound solutions of the Master equation in sharp contrast to soliton perturbation theory and the perturbation of solutions of (2). For example, an amplitude change of a soliton cannot be accommodated by a bound solution but requires contributions of the unbound "continuum". The pulses in the all-fiber soliton laser clearly show this radiation traveling away from the soliton, as shown in Fig. 9. The Kelly sidebands [77] are a manifestation of a phase-matched excitation of these unbound solutions. In contrast, the stretched-pulse Master equation does not predict continuum generation. Experimentally, no clear evidence of Kelly sidebands has emerged in stretched-pulse lasers.

Since the Master equation is an approximate equation, one may ask whether the prediction of the absence of sidebands 


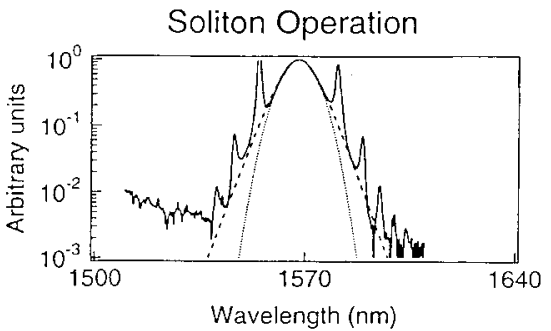

Non-Soliton Operation (Stretched-Pulse)

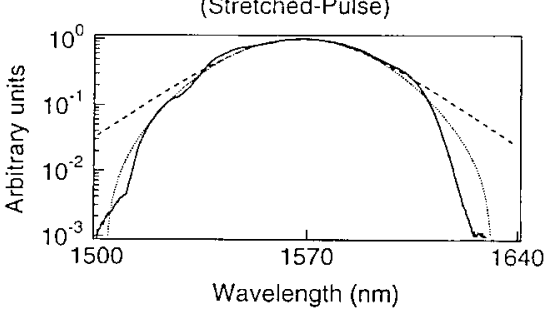

Fig. 12. Comparison of the spectra from the soliton and stretched-pulse lasers. Both spectra are plotted on log scales and fit with the spectra of a sech pulse (dashed) and a Gaussian pulse (dotted)

is an artifact of the approximation. There is another entirely independent argument that shows that sideband generation in a stretched-pulse laser must be much weaker than in a soliton laser. We have argued above that the Gaussian character of the pulses is well realized near the center of the pulse. The structure at the center of the pulse is Fourier transformed into the wings of the spectrum. Thus, the wings of the spectrum should have a decay that is exponential with the square of frequency. This is confirmed experimentally as shown in Fig. 12. In contrast, the spectrum of the pulse solutions of (2) have an exponential decay that is linear with frequency. If the phasematching argument for the generation of Kelly sidebands is applied to the Gaussian spectrum, it is found that the spectral wings that feed the sideband generation are much weaker in the stretched-pulse case than in the soliton case.

The theory of the stretched-pulse laser described in this section considers the nonlinearity and dispersion imbalance to be perturbations. As described in the following sections, the stretched-pulse laser can be scaled to higher powers through increased gain and pump power and larger net positive dispersion. When the nonlinearity is no longer small compared with the linear dispersion, a shift of the minimum pulse-width position in the laser can occur and may decrease the nonlinearity $[114,115]$. The assumption of Gaussian pulses also may lose validity as the power is scaled up. In the following sections, the high-power pulses are generally compared with the hyperbolic-secant profile, which is commonly used in the literature.

\subsection{Pulse dynamics in the stretched-pulse laser}

The pulse in a stretched-pulse laser undergoes large changes in width as well as spectral changes as it circulates the ring. These intracavity pulse dynamics were experimentally investigated in [44] to determine the optimum location for the output coupler and P-APM polarizer. A setup similar to that of Fig. 13 was used, except for the addition of three $3.3 \%$ output couplers constructed of dispersion-shifted fiber and

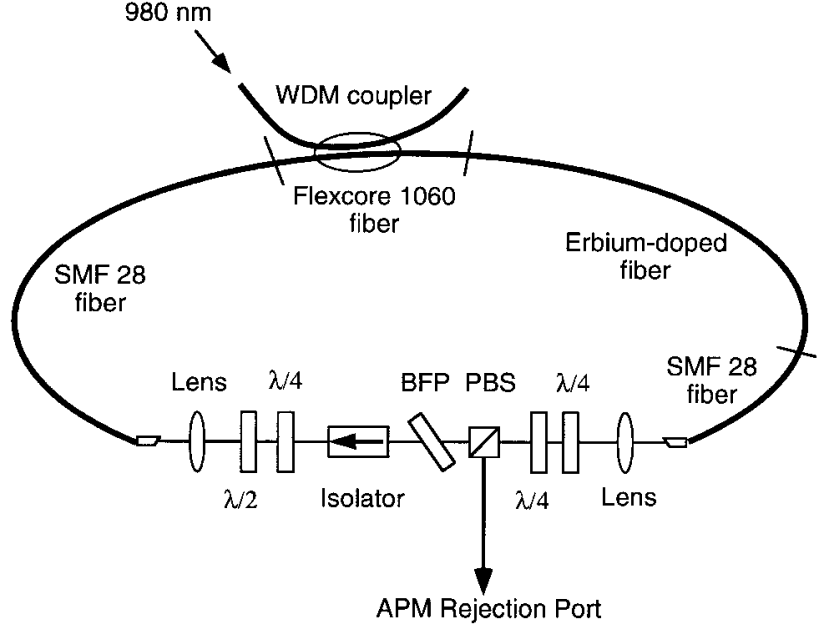

Fig. 13. Experimental setup for stretched-pulse fiber laser with bulk components. For the pulse dynamics study, three 3.3\% dispersion-shifted fiber output couplers were added to the ring immediately before the air gap, immediately after the air gap, and between the WDM coupler and SMF-28 fiber. The rejected $s$ polarization of the PBS acted as the rejection port for the P-APM, giving a linearly polarized output pulse. Reference [43] did not include the birefringent tuning plate

placed immediately before the air gap, after the air gap, and between the WDM coupler and SMF-28 fiber. The laser could be operated in the forward direction indicated by Fig. 13, as well as in the reverse direction by reversing the order of the components in the air gap. The pulses at the three output ports were then investigated for the forward and reverse directions of operation and for varying cavity dispersions (by changing the SMF-28 fiber length with a fixed $\mathrm{Er}^{+3}$-doped fiber length of $1.08 \mathrm{~m}$ ).

In both directions of operation the optimum point for output coupling was immediately after the amplifier, since the pulses had the broadest bandwidth and highest energy. With a net cavity dispersion $D_{\mathrm{T}} \sim+0.009 \mathrm{ps}^{2}$, in reverse operation the power at the $3.3 \%$ output port after the amplifier was $1.55 \mathrm{~mW}$, and the pulses were compressed to $103.3 \mathrm{fs}$ (assuming a Gaussian profile) with TBP of 0.63. Detrimental pulse shaping and structure on the spectrum were attributed to soliton effects, which occurred due to high powers in the negative-dispersion fiber because it was located immediately after the gain fiber. A large loss in bandwidth occurred across the air gap due to the selective rejection of the pulse wings, which contained the spectral extrema.

In forward operation (with $D_{\mathrm{T}} \sim+0.009 \mathrm{ps}^{2}$ ) the pulse spectra were smooth at all three output ports and were not highly sensitive to pump power or waveplate positions. After the amplifier the power at the $3.3 \%$ output port was $0.93 \mathrm{~mW}$, and the de-chirped pulse was $85 \mathrm{fs}$ (assuming Gaussian) with TBP of 0.50 . The spectra before and after the SMF-28 fiber were nearly identical for forward operation, indicating that the propagation was essentially linear through the negativedispersion fiber. The high quality and small wing structure of the de-chirped pulses is due to the highly linear chirp as a result of the normal GVD and SPM in the $\mathrm{Er}^{+3}$-doped fiber as in fiber-grating pair pulse compression [112]. These results clearly indicate that when the pulse stretching per pass is large, optimum pulse characteristics are obtained by minimizing soliton effects. Thus the stretched-pulse laser should be operated in the forward direction with the output port and 
APM polarizer placed immediately after the gain fiber to keep the regions of highest intensities within the positive GVD fiber.

The scalability of the stretched-pulse laser to longer cavity lengths was also addressed in [44]. Although long cavity lengths cause multiple-pulse operation in soliton lasers, that tendency is reduced in the stretched-pulse laser due to operation with high powers in the positive GVD fiber and low powers in the negative GVD fiber. By increasing the $\mathrm{Er}^{+3}$ doped fiber length to $1.55 \mathrm{~m}$ it was found that the output pulse quality was relatively insensitive to cavity length. Pulses as short as 63 fs were achieved with a TBP of 0.60. (In this case, a sech profile was assumed due to soliton compression effects in the fiber dispersive delay line resulting in spectral broadening.) Further scalability is discussed in Sect. 4.5.

\subsection{High energy port}

An important technique for obtaining higher output powers from the stretched-pulse laser was first reported in [43]. In the stretched-pulse laser of Fig. 8 the 10/90 fiber coupler provides a (nonoptimized) 10\% output port, and the laser efficiency is limited by internal losses. A fiber laser mode-locked by P-APM is a nonlinear interferometer with two output ports formed by the final polarizer. The right- and left-hand circular polarizations are added at this polarizer such that the peak of the pulse passes through the polarizer, while the wings of the pulse are in the orthogonal polarization and are extinguished by the polarizer. In the laser of Fig. 8 the polarizer at the isolator input performs this function, and so the rejected polarization is absorbed. Through the use of bulk components, as shown in Fig. 13, the APM rejection port can be accessed as the output port of the stretched-pulse laser. This improves the laser performance by reducing intracavity loss (by eliminating the normal output coupler). Although one might expect the power exiting this port to have poor pulse quality, the rejected pulse can still be of relatively high quality if the differential nonlinear phase shift required for mode-locking is small $(<\pi / 2)$, as demonstrated in [43]. The rejected pulse is usually longer than the intracavity pulse, but the rejected output power can be as much as $50 \%$ of the intracavity power when the laser is biased to a point where there is large loss for low intensities.

The laser consisted of $1.0 \mathrm{~m}$ of $\mathrm{Er}^{+3}$-doped fiber, $2.5 \mathrm{~m}$ of SMF-28 fiber, and $0.45 \mathrm{~m}$ of Corning Flexcor 1060 fiber (with GVD of $-0.007 \mathrm{ps}^{2} / \mathrm{m}$ ) such that the net cavity dispersion was estimated to be $+0.016 \pm 0.005 \mathrm{ps}^{2}$. Self-starting modelocked operation was obtained with $270 \mathrm{~mW}$ of coupled 980-nm pump power and $23.5 \mathrm{~mW}$ of output power. A maximum output power of $38 \mathrm{~mW}(0.78 \mathrm{~nJ}$ pulse energy) was obtained for $340 \mathrm{~mW}$ of coupled pump power. The loss at the PBS (APM rejection port) was estimated to be approximately $50 \%$ of the power circulating in the cavity during pulsed operation, regardless of the wave-plate settings. The high positive chirp on the output pulses was compensated with an SMF28 fiber dispersive delay line, although to demonstrate the chirp compensation only $1 \mathrm{~mW}$ of the laser power was used in order to avoid nonlinear effects in the fiber. The shortest compressed pulse width was $83 \mathrm{fs}$, assuming a sech profile, and the spectral width was $60 \mathrm{~nm}$, corresponding to a timebandwidth product (TBP) of 0.62 .
In reverse operation, self-starting mode-locked operation was observed with $270 \mathrm{~mW}$ of coupled pump power and $23 \mathrm{~mW}$ of output power ( $0.4 \mathrm{~nJ}$ pulse energy). These pulses had high negative chirp, which was compensated by a pair of silicon slabs. Compressed pulses as short as $91 \mathrm{fs}$ were achieved, assuming a sech profile, with $53 \mathrm{~nm}$ of spectral width and a corresponding TBP of 0.59 . When the pump power was increased to obtain higher output powers, the pulse autocorrelations and spectra exhibited multiple peaks and complex structure due to soliton effects. The output pulses from both the forward and reverse directions of operation exhibited larger wings than those observed with standard output coupling as in [42]. Pulse quality was evaluated by comparing the compressed autocorrelations with the ideal sech fit. For the reverse-operation pulse, $\sim 10 \%$ of the total energy was contained in the non-sech component of the pulse, while $\sim 20 \%$ of the total energy of the forward-operation pulse was contained in the non-sech component. Although the reverseoperation pulse exhibited less energy in the non-sech component of the pulse, the limit on output power set by soliton effects indicated that to achieve the highest output powers, forward operation should be used.

4.4.1 Stretched-pulse laser with prisms. Output power levels from the stretched-pulse laser were further increased by replacing the Ti:sapphire pump laser with a commercial 980-nm MOPA [116]. Using the same setup as in Fig. 13 with $1.3 \mathrm{~m}$ of $\mathrm{Er}^{+3}$-doped fiber, average output powers as high as $90 \mathrm{~mW}(2.25 \mathrm{~nJ}$ pulse energy) were obtained from the APM rejection port with $600 \mathrm{~mW}$ of coupled pump power. The chirp on these pulses was then compensated with a double-pass silicon Brewster prism pair to obtain a 90-fs pulse width, assuming a sech profile, with spectral filtering of $\sim 10 \%$ of the pulse energy in the prism compensator to achieve small pulse wings. (Transmission losses through the prism compensator were $\sim 30 \%$ due to clipping of the beam.)

The optimum net dispersion for a stretched-pulse laser using the APM rejection port as the output port was investigated experimentally. As shown in Fig. 14, the negativedispersion fiber was replaced with a pair of Si prisms, allowing the net cavity dispersion $D_{\mathrm{T}}$ to be varied for a fixed length of gain fiber and fixed pulse repetition rate. To obtain higher gain, the $\mathrm{Er}^{+3}$-doped fiber was $1.7 \mathrm{~m}$ and was backward pumped with a 980-nm MOPA. Self-starting, mode-locked

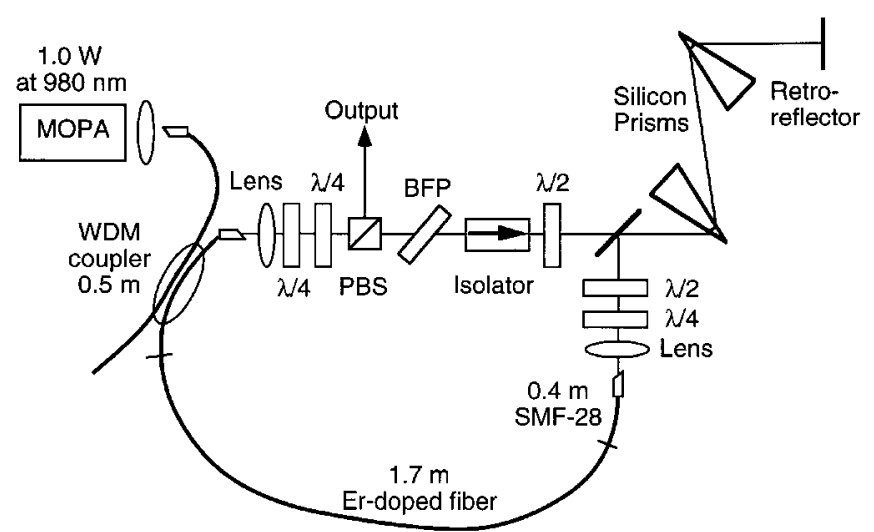

Fig. 14. Experimental setup for a stretched-pulse fiber laser with silicon prisms to provide the negative dispersion 


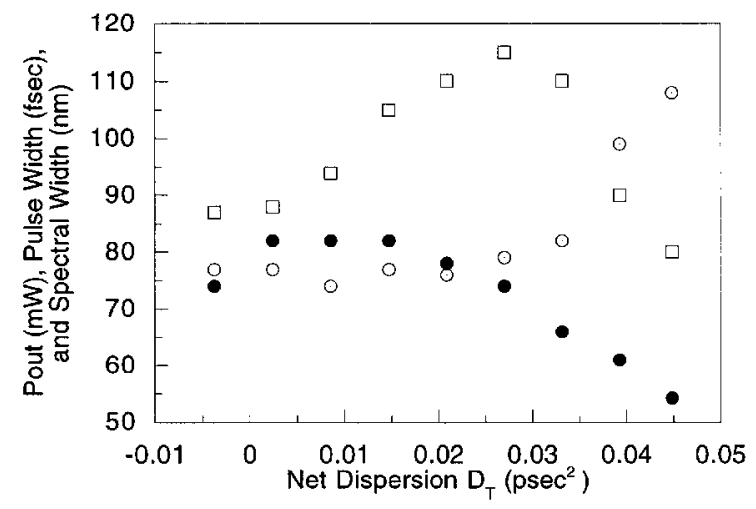

Fig. 15. Plots of average output power $(\square)$, spectral width $(\bullet)$, and compressed pulse width $(\odot)$, versus net cavity dispersion $D_{\mathrm{T}}$ for the stretchedpulse prism laser. The laser output is taken from the APM rejection port. This plot can be compared with Fig. 10, which considered the pulse characteristics from a $10 \%$ output port as a function of $D_{\mathrm{T}}$ by changing the fiber lengths

operation was achieved over a broad range of $D_{\mathrm{T}}$ with output powers as high as $115 \mathrm{~mW}$ directly from the cavity with $520 \mathrm{~mW}$ pump power and $56.3 \mathrm{MHz}$ repetition rate.

While the laser was mode-locked, the prisms could be adjusted to continuously tune $D_{\mathrm{T}}$ from $-0.004 \mathrm{ps}^{2}$ to $+0.045 \mathrm{ps}^{2}$. The average output power, spectral width, and compressed pulse width versus $D_{\mathrm{T}}$ are shown in Fig. 15, where the chirped output pulses were compressed with a 150 lines/mm grating pair instead of a prism pair due to ease of alignment. Slight adjustments to the wave plates and pump power were made at each point to ensure smooth spectra and single-pulse operation. The shortest pulse widths (75 fs) and broadest spectral widths $(80 \mathrm{~nm})$ occurred for $0<D_{\mathrm{T}}<+0.02 \mathrm{ps}^{2}$, while the highest average output power $(115 \mathrm{~mW})$ was achieved for $D_{\mathrm{T}} \approx 0.027 \mathrm{ps}^{2}$. Figure 15 shows similar trends to Fig. 10, which plots the pulse characteristics from a standard $10 \%$ output coupler when the net dispersion was varied by changing the fiber lengths. The results from the stretched-pulse prism laser confirm that optimal output pulses are obtained from either a standard output coupler or the APM rejection port when the net cavity dispersion is slightly positive.

\subsection{Frequency Doubling of the Stretched-Pulse Laser}

In addition to high output power, the stretched-pulse laser has several key features that have led to a number of applications. Synchronized multichannel wavelength-division multiplexing [19] takes advantage of the broad bandwidth of over $50 \mathrm{~nm}$ as would optical characterization applications between 1.5 and $1.6 \mu \mathrm{m}$. The sub-100 fs pulses at $1.55 \mu \mathrm{m}$ have been used to seed a color-center amplifier [22] and may also be used in time-resolved spectroscopy. An additional application, frequency doubling to $775 \mathrm{~nm}$, takes advantage of the high peak power of the stretched-pulse laser. Results indicate that the frequency-doubled stretched-pulse laser is a possible inexpensive and compact replacement for the argon-pumped mode-locked Ti:sapphire oscillator as a seeding source for regenerative amplifiers.

A stretched-pulse laser was optimized for this frequencydoubling application and several nonlinear crystals were eval-

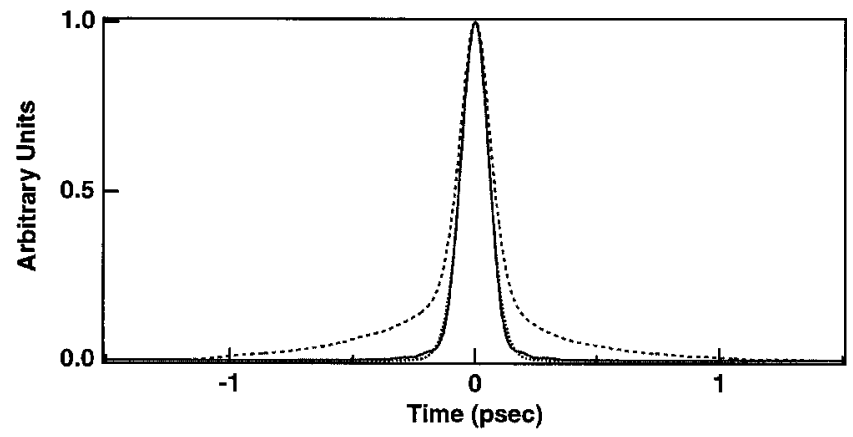

Fig. 16. Autocorrelation of the $1.55-\mu \mathrm{m}$ pulse (dashed) and the frequencydoubled pulse (solid). A sech fit of the frequency-doubled pulse is also shown (dotted), from [45]

uated [45]. The experimental setup was similar to Fig. 13 except that to obtain higher efficiencies and peak power, the $\mathrm{Er}^{+3}$-doped fiber was $1.7 \mathrm{~m}$ (resulting in a larger singlepulse energy) and was backward-pumped with a 980-nm MOPA. The net dispersion $D_{\mathrm{T}}$ of the laser was adjusted to $\approx+0.013 \mathrm{ps}^{2}$ in order to maximize the fundamental pulse energy while maintaining the pulse width near $100 \mathrm{fs}$ [111]. Average output powers of $>95 \mathrm{~mW}$ at $31.8 \mathrm{MHz}$ were obtained with $400 \mathrm{~mW}$ of coupled pump power. The highly chirped $\sim 1 \mathrm{ps}$ pulses were compensated in a sequence of four $\mathrm{Si}$ Brewster prisms with prism separation of $\sim 12 \mathrm{~cm}$ and total transmission of as much as $90 \%$, resulting in fundamental pulse energies of $2.7 \mathrm{~nJ}$. The compressed pulse shown in Fig. 16 was $106 \mathrm{fs}$ (assuming sech) with spectral width of $\sim 67 \mathrm{~nm}$ centered at $1552 \mathrm{~nm}$ and corresponding TBP $\sim 0.89$. Strong pumping and nonlinear output coupling at the APM rejection port caused the fundamental pulses to have excess energy in the pulse wings and larger TBPs than when the stretched-pulse laser was optimized for minimum pulse width [111]. The frequency-doubling results, though, showed that the SHG conversion efficiency was still significantly enhanced by this higher-power operation without comparable conversion of the pulse wing energy. As shown in Fig. 16, the frequency-doubled pulses were clean with less than $1 \%$ of the pulse energy in the non-sech wings.

Several nonlinear crystals including $\beta-\mathrm{BaB}_{2} \mathrm{O}_{4}(\mathrm{BBO})$, $\mathrm{KNbO}_{3}$ (potassium niobate), and $\mathrm{LiB}_{3} \mathrm{O}_{5}$ (LBO) were evaluated for frequency-doubling. Calculations indicate that $\mathrm{BBO}$ should be an excellent nonlinear crystal for doubling $1.55 \mu \mathrm{m}$, because the group velocity mismatch between the fundamental and second harmonic generation (SHG) approaches zero near $1.5 \mu \mathrm{m}$, according to the Sellmeier equations from [117]. With $85 \mathrm{~mW}$ of compressed fundamental power focused onto a 1-cm antireflection (AR)coated BBO crystal with an AR-coated 75-mm focal-length lens, frequency-doubled powers as high as $8.7 \mathrm{~mW}$ were achieved, corresponding to $10 \%$ conversion efficiency and pulse energies of $270 \mathrm{pJ}$. Figure 16 shows the autocorrelation of the $86 \mathrm{fs}$ frequency-doubled pulse (assuming $\mathrm{sech}$ ). The frequency-doubled spectrum is $7.3 \mathrm{~nm}$ wide and centered at $771 \mathrm{~nm}$, resulting in a TBP of 0.32 . Efficient frequency doubling thus results in a large reduction of excess bandwidth to generate near-transform-limited red pulses. Due to the high index of silicon, the compressed pulse beam quality and SHG conversion efficiency 
depended critically on matched prisms and prism alignment.

Although frequency doubling with a $1.5-\mathrm{mm}$ potassium niobate crystal and a 7-mm LBO crystal resulted in conversion efficiencies of $3 \%$ and $6 \%$, respectively, spectral narrowing occurred in both crystals due to group velocity mismatch. The 270-pJ pulses achieved with the 1-cm BBO crystal are sufficient for seeding a Ti:sapphire amplifier, where dominance over amplified spontaneous emission has been demonstrated with 100-pJ seed pulses [118]. Additionally, the $>30 \mathrm{MHz}$ repetition rate of the frequency-doubled stretchedpulse laser results in an easily visible red beam, which simplifies alignment of the amplifier system.

4.5.1 Frequency-resolved optical gating measurements. The offset of the frequency-doubled pulse spectrum from the center of the fundamental spectrum was explored by performing (FROG) measurements $[119,120]$ on both the fundamental and frequency-doubled pulses. Experimental data was taken by spectrally resolving the autocorrelation of the pulse (SHG FROG [121]). Figure 17 shows an experimental SHG FROG trace of a 72-fs fundamental pulse (compressed with a 150 lines/mm grating pair) along with the best calculated fit, obtained with the generalized projections algorithm [122]. The FROG trace indicates that the pulse wings contained the lower-frequency components, and thus efficient frequency doubling occurred at shorter wavelengths. This explains why the frequency-doubled spectrum was centered at $771 \mathrm{~nm}$ rather than at the expected wavelength of $776 \mathrm{~nm}$ based on the first moment of the fundamental spectrum.

The pulse intensity and phase profiles were then calculated from the calculated FROG fit of Fig. 17. The nearly flat phase across the central region of the pulse indicated that
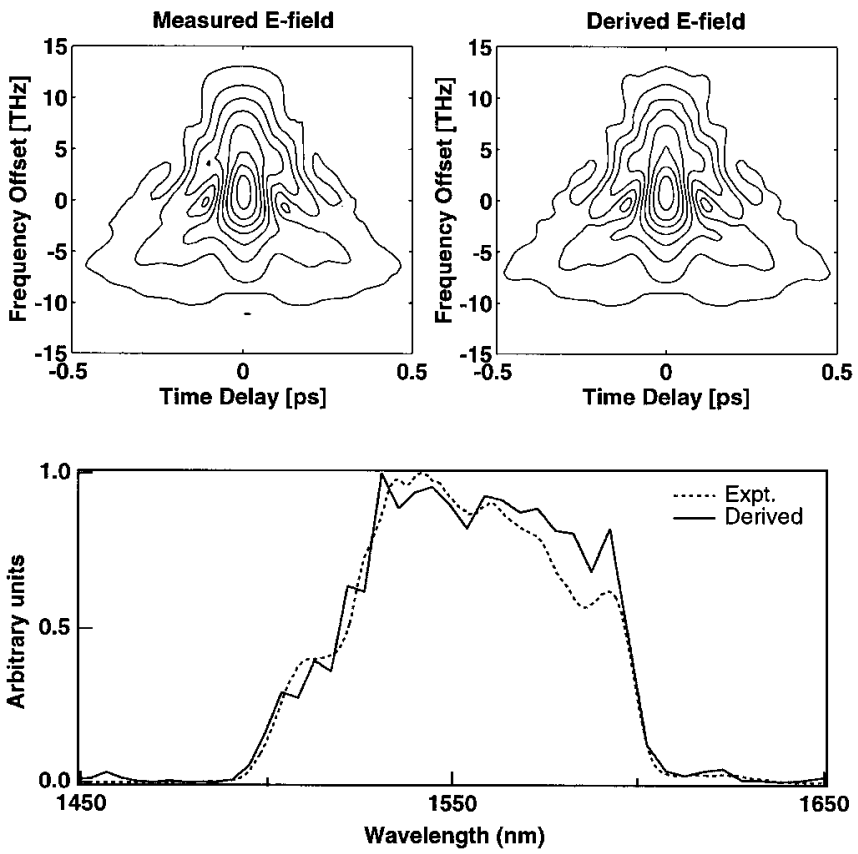

Fig. 17. SHG FROG traces of the fundamental pulse from the stretchedpulse laser: (top left) experimental trace and (top right) calculated fit, from [45]. Bottom plot: comparison of the experimentally measured 1.55- $\mu \mathrm{m}$ spectrum (dashed) with the derived spectrum from the SHG FROG measurement (solid)
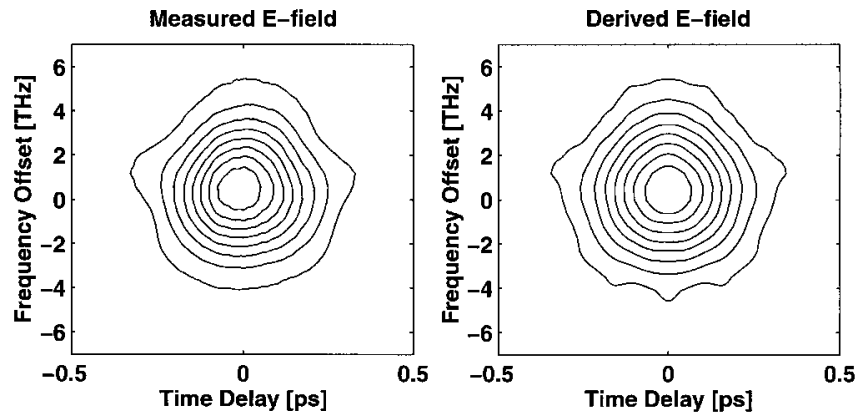

Fig. 18. SHG FROG traces of the frequency-doubled pulse. The contour plot on the left shows the experimentally measured trace, while the right-hand plot shows the calculated fit

the linear chirp was almost completely compensated [45]. In order to confirm that the FROG data accurately represented the pulse, the experimental $1.55-\mu \mathrm{m}$ spectrum was compared with the Fourier transform of the calculated pulse intensity and phase. As shown in Fig. 17, the agreement between the two spectra was excellent, verifying that no filtering occurred in the grating compressor or in the nonlinear crystal. SHG FROG measurements were also performed on the frequency-doubled pulses and are shown in Fig. 18. The round and smooth FROG trace along with the flat calculated phase profile corroborate the high pulse quality of the frequency-doubled output.

\section{Noise in P-APM Fiber Lasers}

Understanding of the noise characteristics of fiber lasers mode-locked by P-APM is important for their potential applications. The long upper-state lifetime of erbium $(\approx 10 \mathrm{~ms})$ causes the $\mathrm{Er}^{+3}$-doped fiber ring laser to be largely insensitive to pump noise and gain fluctuation except at very low frequencies. Thus the laser is well-suited for noise study. Amplitude noise and timing jitter were measured for both the soliton laser and stretched-pulse laser using the method of von der Linde [123], which determines the noise characteristics from the power spectrum of the current of a photodetector when illuminated with the output pulse train. As shown by [123], timing jitter grows quadratically with the harmonic $k$, while amplitude noise is independent of harmonic number.

The noise characteristics of the all-fiber P-APM laser shown in Fig. 2 were examined in [124, 125]. In some modes of operation, and particularly at high pump powers, a cw background would co-propagate with the pulse and appear as a cw spike on the spectrum. The laser would then exhibit relaxation oscillations that were negligible when the $\mathrm{cw}$ spikes were suppressed [124]. The pulse mode and the cw relaxation oscillations were decoupled to the extent that the relaxation frequency was unaffected by the presence of the pulses. Operation with $\mathrm{cw}$ background caused increased timing jitter, with jitter of 14.4 ps observed in a 25 -ms measurement time when the laser had multiple cw spikes compared to jitter as low as $240 \mathrm{fs}$ when the cw spikes were suppressed. The authors also reported that spectral sidebands due to continuum generation did not seem to increase the jitter.

Reference [125] reviewed the Haus-Mecozzi theory [69] of timing jitter and amplitude fluctuations in a mode-locked 
laser with negative GVD. The equations of motion for the different noise components - energy, timing, and frequency - were derived, and the spontaneous emission noise (quantum noise) was modeled by a white noise source. The timing jitter spectrum was found to correspond to a random walk, explaining why free-running passively mode-locked lasers have large timing jitter at low frequencies. Experimental results from the all-fiber soliton ring laser confirmed the theoretical predictions when the cw spike was suppressed. Two sources of timing jitter were identified: noise due to the pump and noise due to spontaneous emission. The measured timing jitter of $640 \mathrm{fs}$, corresponding to $\sim 27 \mathrm{ppm}$ of the pulse roundtrip time, was very close to the quantum limit of $20 \mathrm{ppm}$ (in a measurement time of $0.1 \mathrm{~s}$ ), indicating that the jitter was mainly due to amplified spontaneous emission.

For the stretched-pulse laser Namiki and Haus [126] have proposed a theoretical model of the noise that uses perturbation theory and is analogous to the noise theory for the mode-locked fiber soliton laser [125]. The steady-state pulse within the laser is assumed to be a chirped Gaussian, and the perturbation of the pulse parameters (amplitude, width, phase, chirp, timing, and carrier frequency) is expanded in terms of a complete set of Hermite-Gaussian functions. Fluctuations of the pulse parameters are then determined under the assumption that the noise is due to spontaneous emission of the amplifier. Measurements of an all-fiber stretched-pulse laser (with similar configuration to that of Fig. 8) have been reported in [127]. Amplitude fluctuations as low as $0.05 \%$ of the pulse energy and timing jitter $<80 \mathrm{fs}$ ( $4 \mathrm{ppm}$ of the pulse round-trip time) in a measurement time of $0.09 \mathrm{~s}$ were observed with no external stabilization. Comparison of the measurements with the theory indicates good agreement and also shows that the timing jitter at frequencies $>60 \mathrm{~Hz}$ is due to amplified spontaneous emission (quantum) fluctuations. The decreased timing jitter of the stretched-pulse laser compared to the soliton laser (4 ppm versus $27 \mathrm{ppm}$ ) is caused by the higher power of the stretched-pulse laser and by the smaller net GVD of the stretched-pulse laser, which prevents frequency deviations from being transformed into timing jitter.

These noise levels are the lowest reported to date for passively mode-locked lasers [128-135]. (Valid comparison of the timing jitter of different lasers relies on use of the same measurement time.) Measurements of noise in passively mode-locked fiber lasers have also been reported in [136138], where timing jitter as low as $110 \mathrm{fs}$ rms in the frequency band $30 \mathrm{~Hz}$ to $100 \mathrm{kHz}$ was reported in [136] for a fiber laser with a repetition rate stabilized by a phase-locked loop. These fiber lasers cannot be directly compared with [125, 127] due to use of the $\mathrm{Nd}^{+3}$-doped gain fiber in [136] and semiconductor saturable absorber mode-locking in $[137,138]$.

\section{Conclusion}

Erbium-doped fiber ring lasers mode-locked by P-APM are compact, diode-pumped, stable sources of ultrashort pulses at $1.55 \mu \mathrm{m}$. The lasers can operate in the soliton regime, where the group velocity dispersion is negative, or in the stretched-pulse regime, where sections of both positive- and negative-dispersion fiber are used. In both types of lasers, the ring geometry reduces the self-starting threshold by de- creasing mode pulling due to spurious reflections and spatial hole burning. Although there are inherent limits to the soliton regime due to spectral sideband generation, mode-locked fiber soliton lasers with intracavity filters have produced pulses that are tunable over $50 \mathrm{~nm}$ with durations less than $400 \mathrm{fs}$. The stability region of pulses in a harmonically mode-locked fiber ring laser with soliton pulse compression was mapped, and pulse widths a factor of 4.4 shorter than those predicted by active mode-locking theory were achieved. In the stretched-pulse laser the pulses are alternatively stretched and compressed as they circulate the ring, thus lowering the net nonlinear phase shift per pass. Sub-100-fs pulses with energies as high as $2.7 \mathrm{~nJ}$ and bandwidths $>65 \mathrm{~nm}$ have been generated. The short pulse width, broad bandwidth, and high power of the stretched-pulse laser have led to its application in wavelength-division multiplexing and spectroscopy and as an amplifier seed source. Excellent noise characteristics of both the soliton laser and stretched-pulse laser make them particularly attractive sources.

Acknowledgements. The authors thank GTE Laboratories for donating the Er-doped fiber for the soliton laser and AT\&T for donating the Er-doped fiber for the stretched-pulse lasers. We thank K.L. Hall for help with the harmonic mode-locking experiment and S.B. Fleischer for assistance with the stretched-pulse prism laser and frequency doubling. This research was supported in part by the U.S. Air Force Office of Scientific Research under contract F49620-95-1-0435, the National Science Foundation under contract ECE 94-23737, and the Joint Services Electronics Program under contract DAAH04-95-1-0038. L.E. Nelson gratefully acknowledges fellowship support from the Joint Services Electronics Program.

\section{References}

1. E. Snitzer: Phys. Rev. Lett. 7, 444 (1961)

2. E. Snitzer: In Third International Conference on Quantum Electronics, ed. by P. Grivet, N. Bloembergen: (Columbia University Press 1963) p. 999

3. C. J. Koester, E. Snitzer: Appl. Opt. 3, 1182 (1964)

4. J. Stone, C. A. Burrus: Appl. Phys. Lett. 23, 388 (1973)

5. S. B. Poole, D. N. Payne, R. J. Mears, L. Reekie, M. E. Fermann, R. J. Laming: J. Lightwave Technol. 22, 159 (1986)

6. R. J. Mears, L. Reekie, S. B. Poole, D. N. Payne: Electron. Lett. 22, 159 (1986)

7. J. Y. Allain, M. Monerie, H. Poignant: Electron. Lett. 27, 1513 (1991)

8. R. M. Percival, D. Szebesta, S. T. Davey, N. A. Swain, T. A. King: Electron. Lett. 28, 2064 (1992)

9. D. C. Hanna, I. M. Jauncey, R. M. Percival, I. R. Perry, R. G. Smart, P. J. Suni, J. E. Townsend, A. C. Tropper: Electron. Lett. 24, 1222 (1988)

10. D. C. Hanna, R. M. Percival, R. G. Smart, A. C. Tropper: Opt. Comm. 75, 283 (1990)

11. W. L. Barnes, J. E. Townsend: Electron. Lett. 26, 746 (1990)

12. D. C. Hanna, R. M. Percival, I. R. Perry, R. G. Smart, P. J. Suni, J. E. Townsend, A. C. Tropper: Electron. Lett. 24, 1111 (1988)

13. H. M. Pask, R. J. Carman, D. C. Hanna, A. C. Tropper, C. J. Mackechnie, P. R. Barber, J. M. Dawes: IEEE J. Selected Topics in Quant. Electron. 1, 2 (1995)

14. Y. Durteste, M. Monerie, J. Y. Allain, H. Poignant: Electron. Lett. 27, 626 (1991)

15. T. Whitley, R. Wyatt, D. Szebesta, S. Davey: IEEE Photon. Tech. Lett. 4, 401 (1993)

16. K. Tamura, E. Yoshida, E. Yamada, M. Nakazawa: Electron. Lett. 32, 835 (1996)

17. R. A. Barry, V. W. S. Chan, K. L. Hall, E. S. Kintzer, J. D. Moores, K. A. Rauschenbach, E. A. Swanson, L. E. Adams, C. R. Doerr, S. G. Finn, H. A. Haus, E. P. Ippen, W. S. Wong, M. Haner: IEEE Journal on Selected Areas in Communications 14, 999 (1996)

18. E. A. DeSouza, M. C. Nuss, M. Zirngibl, C. H. Joyner: In Optical Fiber Communication Conference 1995, San Diego, CA (1995), paper PD16 
19. M. C. Nuss, W. H. Knox, J. B. Stark, S. Cundiff, D. A. B. Miller, U. Koren: In Ultrafast Phenomena X, ed. by P. F. Barbara, J. G. Fujimoto, W. H. Knox, W. Zinth (Springer Berlin 1996) chap. 1, p. 22

20. K. Tamura, E. Yoshida, M. Nakazawa: Electron. Lett. 32, 1691 (1996)

21. M. Hofer, M. H. Ober, F. Haberl, M. E. Fermann, E. R. Taylor, K. P. Jedrzejewski: Opt. Lett. 17, 807 (1992)

22. G. Lenz, W. Gellermann, D. J. Dougherty, K. Tamura, E. P. Ippen: Opt. Lett. 21, 137 (1996)

23. A. Hariharan, M. E. Fermann, M. L. Stock, D. J. Harter, J. Squier: Opt. Lett. 21, 128 (1996)

24. M. E. Fermann, M. Hofer, F. Haberl, A. J. Schmidt, L. Turi: Opt. Lett. 16, 244 (1991)

25. I. N. Duling III: Electron. Lett. 27, 544 (1991)

26. D. J. Richardson, R. I. Laming, D. N. Payne, M. W. Phillips, V. J. Matsas: Electron. Lett. 27, 730 (1991)

27. M. Hofer, M. E. Fermann, F. Haberl, M. H. Ober, A. J. Schmidt: Opt. Lett. 16, 502 (1991)

28. V. J. Matsas, T. P. Newson, D. J. Richardson, D. N. Payne: Electron. Lett. 28, 1391 (1992)

29. K. Tamura, H. A. Haus, E. P. Ippen: Electron. Lett. 28, 2226 (1992)

30. M. Zirngibl, L. W. Stulz, J. Stone, J. Hugi, D. DiGiovanni, P. B. Hansen: Electron. Lett. 27, 1734 (1991)

31. W. H. Loh, D. Atkinson, P. R. Morkel, M. Hopkinson, A. Rivers, A. J. Seeds, D. N. Payne: IEEE Photon. Tech. Lett. 5, 35 (1993)

32. E. A. DeSouza, C. E. Soccolich, W. Pleibel, R. H. Stolen, M. N. Islam, J. R. Simpson, D. J. DiGiovanni: Electron. Lett. 29, 447 (1993)

33. M. Nakazawa, E. Yoshida, Y. Kimura: Electron. Lett. 29, 63 (1993)

34. B. C. Barnett, L. Rahman, M. N. Islam, Y. C. Chen, P. Bhattacharya, W. Riha, K. V. Reddy, A. T. Howe, K. A. Stair, H. Iwamura, S. R. Friberg, T. Mukai: Opt. Lett. 20, 471 (1995)

35. M. H. Ober, M. Hofer, U. Keller, T. H. Chiu: Opt. Lett. 18, 1532 (1993)

36. S. Tsuda, W. H. Knox, J. L. Zyskind, J. E. Cunningham, J. Y. Pan, R. Pathak: In CLEO 1996 Technical Digest Series (1996) Paper CFD2

37. M. E. Fermann, M. J. Andrejco, Y. Silberberg, A. M. Weiner: Opt. Lett. 18, 48 (1993)

38. C. J. Chen, P. K. A. Wai, C. R. Menyuk: Opt. Lett. 17, 417 (1992)

39. M. E. Fermann, M. J. Andrejco, Y. Silberberg, M. L. Stock: Opt. Lett. 18, 894 (1993)

40. M. E. Fermann, K. Sugden, I. Bennion: Opt. Lett. 20, 1625 (1995)

41. K. Tamura, E. P. Ippen, H. A. Haus: IEEE Photon. Tech. Lett. 6, 1433 (1994)

42. K. Tamura, E. P. Ippen, H. A. Haus, L. E. Nelson: Opt. Lett. 18, 1080 (1993)

43. K. Tamura, C. R. Doerr, L. E. Nelson, E. P. Ippen, H. A. Haus: Opt. Lett. 19, 46 (1994)

44. K. Tamura, E. P. Ippen, H. A. Haus: Appl. Phys. Lett. 67, 158 (1995)

45. L. E. Nelson, S. B. Fleischer, G. Lenz, E. P. Ippen: Opt. Lett. 21, 1759 (1996)

46. M. H. Ober, M. Hofer, M. E. Fermann: Opt. Lett. 18, 367 (1993)

47. M. Hofer, M. H. Ober, R. Hofer, M. E. Fermann, G. Sucha, D. Harter, K. Sugden, I. Bennion, C. A. C. Mendonca, T. H. Chiu: Opt. Lett. 20, 1701 (1995)

48. M. H. Ober, M. Hofer, R. Hofer, G. A. Reider, G. D. Sucha, M. E. Fermann, D. Harter, C. A. C. Mendonca, T. H. Chiu: Opt. Lett. 20, 2303 (1995)

49. T. Sugawa, E. Yoshida, Y. Miyajima, M. Nakazawa: Electron. Lett. 29, 902 (1993)

50. M. J. Guy, D. U. Noske, A. Boskovic, J. R. Taylor: Opt. Lett. 19, 828 (1994)

51. L. E. Nelson, E. P. Ippen, H. A. Haus: Appl. Phys. Lett. 67, 19 (1995)

52. R. C. Sharp, D. E. Spock, N. Pan, J. Elliot: Opt. Lett. 21, 881 (1996)

53. J. Mark, L. Y. Liu, K. L. Hall, H. A. Haus, E. P. Ippen: Opt. Lett. 14, 48 (1989)

54. E. P. Ippen, H. A. Haus, L. Y. Liu: J. Opt. Soc. Am. B 6, 1736 (1989)

55. H. A. Haus, J. G. Fujimoto, E. P. Ippen: J. Opt. Soc. Am. B 8, 2068 (1991)

56. P. D. Maker, R. W. Terhune: Phys. Rev. Lett. 12, 507 (1964)

57. R. H. Stolen, J. Botineau, A. Ashkin: Opt. Lett. 7, 512 (1982)

58. E. P. Ippen: In Ultrafast Phenomena VIII, ed. by J. L. Martin, A. Migus, G. A. Mourou, A. H. Zewail (Springer, Berlin 1993) chap. 4 p. 155

59. E. P. Ippen: Appl. Phys. B 58, 159 (1994)

60. F. Krausz, T. Brabec, Ch. Spielmann: Opt. Lett. 16, 235 (1991)

61. K. Tamura, J. Jacobson, E. P. Ippen, H. A. Haus, J. G. Fujimoto: Opt.
Lett. 18, 220 (1993)

62. F. Krausz, T. Brabec: Opt. Lett. 18, 888 (1993)

63. H. A. Haus, E. P. Ippen: Opt. Lett. 16, 1331 (1991)

64. O. E. Martinez, R. L. Fork, J. P. Gordon: Opt. Lett. 9, 156 (1984)

65. O. E. Martinez, R. L. Fork, J. P. Gordon: J. Opt. Soc. Am. B 2, 753 (1985)

66. H. A. Haus, E. P. Ippen, K. Tamura: IEEE J. QE-30, 200 (1994)

67. L. F. Mollenauer, J. P. Gordon, M. N. Islam: IEEE J. QE-22, 157 (1986)

68. L. F. Mollenauer, S. G. Evangelides, H. A. Haus: J. Lightwave Technol. 9, 194 (1991)

69. H. A. Haus, A. Mecozzi: IEEE J. QE-29, 983 (1993)

70. J. D. Moores: Opt. Comm. 96, 65 (1993)

71. D. J. Richardson, R. I. Laming, D. N. Payne, M. W. Phillips, V. Matsas: Electron. Lett. 27, 1451 (1991)

72. A. B. Grudinin, D. J. Richardson, D. N. Payne: Electron. Lett. 28, 67 (1992)

73. A. Hasegawa: Appl. Opt. 23, 3302 (1984)

74. S. M. J. Kelly, K. Smith, K. J. Blow, N. J. Doran: Opt. Lett. 16, 1337 (1991)

75. J. P. Gordon: J. Opt. Soc. Am. B 9, 91 (1992)

76. N. Pandit, D. U. Noske, S. M. J. Kelly, J. R. Taylor: Electron. Lett. 28, 455 (1992)

77. S. M. J. Kelly: Electron. Lett. 28, 806 (1992)

78. K. Tamura, C. R. Doerr, H. A. Haus, E. P. Ippen: IEEE Photon. Tech. Lett. 6, 697 (1994)

79. A. E. Siegman: In Lasers (University Science Books, Mill Valley, CA 1986)

80. I. P. Alcock, A. I. Ferguson, D. C. Hanna, A. C. Tropper: Electron. Lett. 22, 269 (1986)

81. G. Geister, R. Ulrich: Opt. Comm. 68, 187 (1988)

82. D. C. Hanna, A. Kazer, M. W. Phillips, D. P. Shepherd, P. J. Suni: Electron. Lett. 25, 95 (1989)

83. J. D. Kafka, T. Baer, D. W. Hall: Opt. Lett. 14, 1269 (1989)

84. A. Takada, H. Miyazawa: Electron. Lett. 26, 216 (1990)

85. E. Yoshida, Y. Kimura, M. Nakazawa: Appl. Phys. Lett. 60, 932 (1992)

86. G. T. Harvey, L. F. Mollenauer: Opt. Lett. 18, 107 (1993)

87. C. R. Doerr, H. A. Haus, E. P. Ippen, M. Shirasaki, K. Tamura: Opt. Lett. 19, 31 (1994)

88. M. Nakazawa, K. Tamura, E. Yoshida: Electron. Lett. 32, 461 (1996)

89. K. Tamura, M. Nakazawa: Opt. Lett. 21, 1930 (1996)

90. X. Shan, D. Cleland, A. Ellis: Electron. Lett. 28, 182 (1992)

91. M. Nakazawa, E. Yoshida, Y. Kimura: Electron. Lett. 30, 1603 (1994)

92. K. Tamura, M. Nakazawa: Opt. Lett. 24, 1984 (1996)

93. C. R. Doerr, H. A. Haus, E. P. Ippen: Opt. Lett. 19, 1958 (1994)

94. H. A. Haus, D. J. Jones, E. P. Ippen, W. S. Wong: J. Lightwave Technol. 14, 622 (1996)

95. P. V. Mamyshev, L. F. Mollenauer: Opt. Lett. 19, 2083 (1994)

96. D. J. Jones, H. A. Haus, E. P. Ippen: Opt. Lett. 21, 1818 (1996)

97. D. J. Kuizenga, A. E. Siegman: IEEE J. Quantum Electron. 6, 694 (1970)

98. H. A. Haus, Y. Silberberg: IEEE J. Quantum Electron. QE-22, 325 (1986)

99. M. W. Phillips, A. I. Ferguson, D. C. Hanna: Opt. Lett. 14, 219 (1989)

100. M. Hofer, M. E. Fermann, F. Haberl, J. E. Townsend: Opt. Lett. 15, 1467 (1990)

101. D. Burns, W. Sibbett: Electron. Lett. 26, 505 (1990)

102. K. Smith, J. R. Armitage, R. Wyatt, N. J. Doran: Electron. Lett. 26, 1149 (1990)

103. R. P. Davey, N. Langford, A. I. Ferguson: Electron. Lett. 27, 726 (1991)

104. F. X. Kartner, D. Kopf, U. Keller: J. Opt. Soc. Am. B 12, 486 (1995)

105. H. A. Haus, A. Mecozzi: Opt. Lett. 17, 1500 (1992)

106. C. R. Doerr, W. S. Wong, H. A. Haus, E. P. Ippen: Opt. Lett. 19, 1747 (1994)

107. K. L. Hall, J. D. Moores, K. A. Rauschenbach, W. S. Wong: IEEE Photonics Technol. Lett. 7, 1093 (1995)

108. J. D. Moores, W. S. Wong, K. L. Hall: Opt. Lett. 20, 2547 (1995)

109. M. E. Fermann, M. J. Andrejco, M. L. Stock, Y. Silberberg, A. M. Weiner: In OSA Annual Meeting, Vol. 23 of 1992 OSA Technical Digest Series (Optical Society of America, Washington DC, 1992), Paper PD16

110. H. A. Haus, K. Tamura, L. E. Nelson, E. P. Ippen: IEEE J. QE-31, 591 (1995)

111. K. Tamura, L. E. Nelson, H. A. Haus, E. P. Ippen: Appl. Phys. Lett. 64, 
149 (1994)

112. W. J. Tomlinson, R. J. Stolen, C. V. Shank: J. Opt. Soc. Am. B 1, 139 (1984)

113. M. Matsumoto, H. A. Haus: Submitted to Opt. Lett.

114. K. Tamura: Additive pulse mode-locked erbium-doped fiber lasers. $\mathrm{PhD}$ thesis, MIT 1994

115. K. Tamura, M. Nakazawa: Appl. Phys. Lett. 67, 3691 (1995)

116. G. Lenz, K. Tamura, H. A. Haus, E. P. Ippen: Opt. Lett. 20, 1289 (1995)

117. D. Eimerl, L. Davis, S. Velsko, E. K. Graham, A. Zalkin: J. Appl. Phys. 62, 1968 (1987)

118. T. B. Norris: Opt. Lett. 17, 1009 (1992)

119. D. J. Kane, R. Trebino: IEEE J. QE-29, 571 (1993)

120. J. Paye, M. Ramaswamy, J. G. Fujimoto, E. P. Ippen: Opt. Lett. 18, 1946 (1993)

121. K. W. DeLong, R. Trebino, J. Hunter, W. E. White: J. Opt. Soc. Am. B 11, 2206 (1994)

122. K. W. DeLong, D. N. Fittinghoff, R. Trebino: Opt. Lett. 19, 2152 (1994)

123. D. von der Linde: Appl. Phys. B 39, 201 (1986)

124. S. Namiki, E. P. Ippen, H. A. Haus, K. Tamura: Appl. Phys. Lett. 69, 3969 (1996)
125. S. Namiki, C. X. Yu, H. A. Haus: J. Opt. Soc. Am. B 13, 2817 (1996)

126. S. Namiki, H. A. Haus: IEEE J. QE-33, 649 (1997)

127. C. X. Yu, S. Namiki, H. A. Haus: IEEE J. QE-33, 660 (1997)

128. U. Keller, K. Li, M. Rodwell, D. Bloom: IEEE J. QE-25, 280 (1989)

129. A. Finch, X. Zhu, P. N. Kean, W. Sibbett: IEEE J. QE-26, 1115 (1990)

130. U. Keller, C. E. Soccolich, G. Sucha, M. N. Islam, M. Wegener: Opt. Lett. 15, 974 (1990)

131. G. T. Harvey, M. S. Heutmaker, P. R. Smith, M. C. Nuss, U. Keller, J. A. Valdmanis: IEEE J. QE-27, 295 (1991)

132. S. B. Darack, D. R. Dykaar: Opt. Lett. 16, 1677 (1991)

133. D. E. Spence, J. M. Evans, W. E. Sleat, W. Sibbett: Opt. Lett. 16, 1762 (1991)

134. D. R. Walker, D. W. Crust, W. E. Sleat, W. Sibbett: IEEE J. QE-28, 289 (1992)

135. J. Son, J. V. Rudd, J. F. Whitaker: Opt. Lett. 17, 733 (1992)

136. F. Haberl, M. H. Ober, M. Hofer, M. E. Fermann, E. Wintner, A. J. Schmidt: IEEE Photon. Tech. Lett. 3, 1071 (1991)

137. D. Abraham, R. Nagar, G. Eisenstein: Opt. Lett. 18, 1508 (1993)

138. L. Rahman, B. C. Barnett, M. N. Islam, H. Lin, R. Fortenberry, K. W. Chang, S. A. Newton: CLEO 1995 Technical Digest Series, Baltimore MD (1995), Paper JTuE2 